\title{
THE
}

\section{Implementation of a Mesh Movement Scheme in a Multiply Nested Ocean Model and Its Application to Air-Sea Interaction Studies}

C. Rowley

University of Rhode Island

Isaac Ginis

University of Rhode Island, iginis@uri.edu

\section{Citation/Publisher Attribution}

Rowley, C., \& Ginis, I. (1999). Implementation of a Mesh Movement Scheme in a Multiply Nested Ocean Model and Its Application to Air-Sea Interaction Studies. Mon. Wea. Rev., 127, 1879-1896. doi: 10.1175/ 1520-0493(1999)1272.0.CO;2

Available at: https://doi.org/10.1175/1520-0493(1999)127<1879:IOAMMS>2.0.C0;2

This Article is brought to you for free and open access by the Graduate School of Oceanography at DigitalCommons@URI. It has been accepted for inclusion in Graduate School of Oceanography Faculty Publications by an authorized administrator of DigitalCommons@URI. For more information, please contact digitalcommons-group@uri.edu. 


\title{
Implementation of a Mesh Movement Scheme in a Multiply Nested Ocean Model and Its Application to Air-Sea Interaction Studies
}

\author{
C. ROWLEY AND I. GINIS \\ Graduate School of Oceanography, University of Rhode Island, Narragansett, Rhode Island
}

(Manuscript received 30 January 1998, in final form 1 September 1998)

\begin{abstract}
A mesh movement scheme is implemented in a multiply nested primitive equation ocean model. Mesh movement can be specified or determined in the course of the model run so as to follow an evolving oceanic feature, such as a wave front or propagating eddy, or atmospheric forcing, such as a tropical cyclone. Mass, heat, and momentum are conserved during the movement. The mesh movement scheme is tested in idealized and realistic configurations of the model. The idealized tests include simulations in which the moving meshes follow a propagating equatorial Kelvin wave, a dipole, or move across an existing mesoscale eddy. The tests demonstrate that the solutions in the fine-mesh region of the nested meshes reproduce well the equivalent solutions from uniform fine-mesh models.

The model is applied for simulations of the ocean response to tropical cyclones, in which the moving meshes maintain high resolution near the cyclone center. The solution in the inner meshes reproduces very well the uniform fine-mesh simulation, in particular the sea surface temperature. It demonstrates that the moving meshes do not degrade the solution, even with the application of strong winds and the generation of energetic surface currents and near-inertial gravity waves.

The mesh movement scheme is also successfully applied for a real-case simulation of the ocean response to Typhoon Roy (1988) in the western North Pacific. For this experiment, the model is initialized using the fields from a general circulation model (GCM) multiyear spinup integration of the large-scale circulation in the tropical Pacific Ocean. The nested-mesh solution shows no difficulty simulating the interaction of the storm-induced currents with the existing background circulation.
\end{abstract}

\section{Introduction}

Nested-mesh numerical models have in recent years become useful research and operational tools for simulating mesoscale meteorological and oceanic phenomena in the large-scale environment. The important advantage of a multiply nested mesh arrangement is that it allows improved horizontal resolution in a limited region without requiring a fine grid resolution throughout the entire model domain. Therefore the model domain to be resolved with higher resolution is kept to a minimum, greatly reducing computer memory and speed requirements, and allowing better resolution than would otherwise be possible.

For many geophysical applications, however, our interest is not so much in a limited region as it is in a particular process. Moving weather systems, propagating waves, and oceanic mesoscale eddies represent classes of phenomena for which a nested mesh configuration

Corresponding author address: Dr. Clark Rowley, Graduate School of Oceanography, University of Rhode Island, Narragansett, RI 02882 .

E-mail: c.rowley@gso.uri.edu with permanently fixed fine-grid meshes may not be adequate. Moving tropical cyclones are one example of this type of phenomenon. Meteorologists have for some time recognized that simulations of tropical cyclone dynamics require very high resolution to better resolve the storm inner-core structure. Because tropical cyclones usually travel thousands of kilometers, it is not computationally efficient to use fine-grid resolution over the entire area of the storm track. Instead, movable multiply nested mesh configurations are used for hurricane predictions. Atmospheric models with this capability have been in use for the last few decades (e.g., Harrison 1973; Ley and Elsberry 1976; Jones 1977). For example, the official operational hurricane prediction model for the National Weather Service developed at the National Oceanic and Atmospheric Administration (NOAA) Geophysical Fluid Dynamics Laboratory (GFDL; Kurihara et al. 1998) is based on the movable nested-mesh framework. It provides the means to translate the finestscale meshes with the moving hurricane, thus placing the fine resolution exactly where it is needed, in the vicinity of the storm center.

Proper simulation of tropical cyclones calls for the use of fully coupled atmosphere-ocean models. Recent numerical studies using coupled hurricane-ocean mod- 
els (e.g., Khain and Ginis 1991; Bender et al. 1993a; Schade and Emanuel 1999; Falkovich et al. 1995) have demonstrated that interaction with the ocean has an important impact on storm intensity. This is because tropical cyclones moving over the ocean produce significant changes in the sea surface temperature (SST). Observational and numerical studies have shown that the SST may decrease up to $6^{\circ} \mathrm{C}$ underneath a tropical cyclone as a result of the strong wind forcing (e.g., see the review article of Ginis 1995). This amount of cooling can significantly reduce the heat and moisture fluxes at the sea surface, which play an important role in storm evolution.

In developing a coupled tropical cyclone-ocean mod$\mathrm{el}$, the same issue of computational efficiency applies to the ocean component of the coupled system: high horizontal resolution is needed only in the region within several radii of the maximum wind. Therefore, a movable nested-mesh configuration is also highly desirable for efficient modeling of the ocean response to tropical cyclone forcing. This capability is especially important for coupled tropical cyclone-ocean simulations in the eastern and western Pacific, because it is not computationally practical to use a single very high resolution mesh over the entire Pacific Ocean. However, all of the few recently developed nested-mesh ocean models utilize mesh configurations that are fixed in time and space (e.g., Spall and Holland 1991; Oey and Chen 1992; Fox and Maskell 1995; Ginis et al. 1998) and, therefore, are not very suitable for simulations of the ocean response to a moving storm.

The purpose of this paper is to present the implementation of a mesh movement scheme in a nested-mesh primitive equation ocean model, and the results of its rigorous testing in idealized and realistic ocean settings. The model is designed to allow the inner meshes of finer resolution to follow moving oceanic or atmospheric features. It employs a mesh movement technique originally proposed by Kurihara et al. (1979) and successfully applied for many years in the GFDL hurricane prediction model (Kurihara et al. 1998). Although the movement scheme has been implemented in the model with the coupled tropical cyclone-ocean application in mind, we envision its use in a variety of studies that focus on moving mesoscale ocean and coupled air-sea variability.

The mesh movement scheme is implemented here in the nested-mesh ocean circulation model recently developed by Ginis et al. (1998). A distinguishing characteristic of their model is the method by which regions of fine resolution are embedded into a more coarsely resolved domain. The model employs a two-way interactive nesting technique and includes the following features: 1$)$ the interface where the two integration domains dynamically interact with each other is separated from the change in resolution at the mesh interface; 2) the interaction at the dynamical interface is expressed in the form of a flux condition, so that conservation properties of mass, momentum, and heat are satisfied everywhere;
3) the formulation of the mesh nesting algorithm allows flexibility in deciding the number of meshes and the ratios of grid resolutions between adjacent meshes. If the grid ratio is made to be unity, the model simply reduces to a single mesh configuration and can be used as a large-scale general circulation model (GCM).

In the next sections of this paper, the formulation of the movement scheme is presented, as well as various simulations designed to test the method and to demonstrate its utility for certain types of geophysical problems. A brief outline of the model physics and a description of the mesh nesting technique are presented in section 2, and a detailed discussion of the mesh movement method in section 3. In section 4 results from idealized cases used to test the mesh movement technique are described. Section 5 presents realistic simulations that demonstrate the application of the movable nested-mesh method to simulations of the ocean response to tropical cyclone forcing. We conclude with a summary discussion in section 6 .

\section{Nested mesh ocean model}

A brief outline of the model physics and numerics is included here for clarity, with a review of the key principles of the mesh nesting technique. For a full description of the model the reader is referred to Ginis et al. (1998).

\section{a. Model physics and numerics}

The model is based on a primitive equation multilayered formulation in spherical coordinates. The mixed layer is treated as a turbulent boundary layer that exchanges momentum and heat with the atmosphere at its surface, and with the thermocline by entrainment at its base. It is well mixed due to turbulent mixing and is vertically homogeneous in density. The stratified thermocline below is divided into an arbitrary number of numerical layers, according to a sigma coordinate system originally proposed by Gent and Cane (1989). The essence of the sigma coordinate is to keep the ratio of the layer depths below the mixed layer equal to a prescribed value. With this vertical coordinate, the layer depths are rearranged at each time step in the course of the model integration. As a result, mass fluxes are introduced across the layer interfaces. The mass fluxes are calculated diagnostically, along with their accompanying fluxes of momentum, heat, and salt.

The effects of horizontal diffusion of momentum, heat, and salt are estimated by the Smagorinsky nonlinear viscosity scheme (Smagorinsky 1963). The vertical diffusion processes include three major mechanisms of vertical turbulent mixing in the upper ocean, that is, wind stirring, shear instability, and convective overturning. The parameterization of these processes is based on the hybrid mixing scheme of Chen et al. (1994). The version of the model used in this study 
employs the reduced gravity assumption so that the deep ocean is at rest below the active upper ocean.

A nonstaggered grid system is used in which all model variables are calculated at the middle of the grid cells. Finite differencing of the governing equations is based on the box method originally developed by Bryan (1966) and Kurihara and Holloway (1967) and adopted for the nested-mesh configuration by Kurihara et al. (1979). The time integration is performed with the twostep iteration scheme of Kurihara and Tripoli (1976). It is designed to preserve low-frequency, slow modes while suppressing high-frequency, fast oscillatory modes. The scheme uses weights, $\alpha$ and $\beta$, for the lowfrequency advective terms, and for the high-frequency Coriolis and pressure gradient terms, respectively. Kurihara and Tripoli (1976) suggested using weights of $\alpha$ $=0.506$ and $\beta=1$, but later Kurihara and Bender (1980) reported improved results using $\alpha=0.506$ and $\beta=2.5$, and these values were implemented in the experiments in Ginis et al. (1998). However, it was determined in the present study that in certain model applications, a value of $\beta=2.5$ may too strongly damp some important physical modes, especially for the larger time steps used in the coarser meshes. Therefore, for most of the experiments presented here, a smaller value of $\beta=0.506$ was successfully applied. In addition to the use of this frequency-selective time-integration method, nonlinear computational instability in this model is effectively suppressed by the occasional application of a spatial smoothing-desmoothing technique described in Bender et al. (1993b). The frequency of application of the smoothing-desmoothing is typically once every 18 or 24 time steps, when all velocity and mass fields are treated.

\section{b. Mesh nesting}

The model uses the nested-mesh technique originally proposed in Kurihara et al. (1979), which embeds meshes of fine resolution into a more coarsely resolved domain using a two-way interactive nesting technique. In this method, special care is taken along the interfaces to implement the two-way interactions of the adjacent meshes. The dynamical interfaces, where fluxes pass between the integration domains of differing time steps, are kept separate from the mesh interfaces, where the horizontal resolution changes from one mesh to the next. Figure 1 shows a schematic of a portion of a typical mesh configuration, for a case with three meshes where the grid cell sizes are in the ratio 1:2:4. The time steps for the integration domains are in the same ratio as the grid resolutions. Note that the integration domains $\mathrm{A}$, $\mathrm{B}$, and $\mathrm{C}$ are distinguished from the corresponding meshes 1, 2, and 3. For each inner mesh, two surrounding rows or columns of grid points from the next coarser mesh are integrated forward in time with the inner mesh gridpoints. These two rows, called a window frame, separate the mesh interfaces, where noise is likely to be

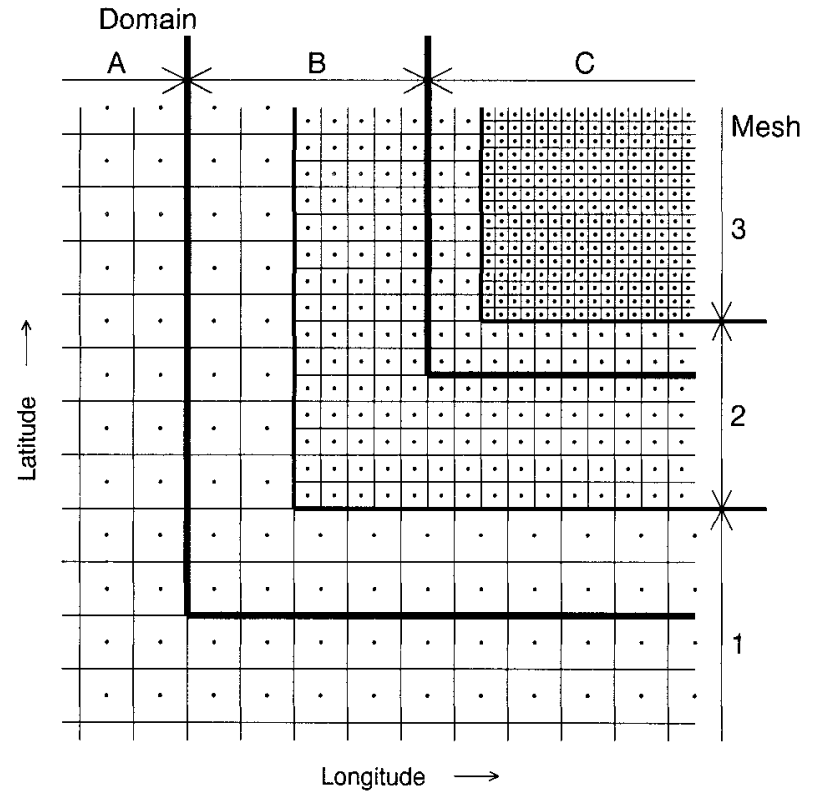

FIG. 1. A schematic example of a portion of a nested-mesh configuration. The integration domains are indicated by the letters A, B, C. The meshes are indicated by the numbers $1,2,3$. The heavy dark lines indicate the dynamical interfaces, and the medium-weight lines show the mesh interfaces. All variables are located at the centers of the cells, indicated by the dots in this schematic. In this example, the grid spacing is in the ratio $1: 2: 4$.

due to the change in resolution, from the interfaces between the integration domains, where noise may be generated by the dynamical coupling.

The order of forward integration for the different domains is based on two rules. First, each domain is integrated only after all interior domains are synchronized with the current domain. Second, when two domains are synchronized, integration proceeds from the outer domain. Using these two rules, the order of integration for the first full time step of the example in Fig. 1 would be as follows: domain A, B, C, C, B, C, C, and this pattern would repeat for subsequent time steps.

The two-way interaction between adjacent domains is illustrated for domains A and B. At each time step, the forward integration begins with the coarser domain A. This integration takes into account the values in the window frame of domain B. During the calculation, the horizontal fluxes and all model variables are preserved along the dynamical interface and used to specify the boundary conditions for the integration of domain B. Because domain B has a smaller time step, the boundary conditions are interpolated in time so that the sums of the fluxes during the integration of domain B exactly equal those obtained for domain $\mathrm{A}$. In this way, fluxes of mass, heat, salinity, and momentum can be conserved at these interfaces.

\section{Mesh movement scheme}

The present model is designed to allow the inner meshes of finer resolution to follow oceanic or atmo- 
spheric mesoscale features. If a simulated oceanic disturbance (or atmospheric forcing) moves during the time integration, the finer-resolution meshes will follow it, thus providing better model forecasts of smaller-scale phenomena. In this section we describe the main principals of the mesh movement algorithm.

At the beginning of a model integration, the inner meshes are positioned with reference to the location of the simulated feature. Movement of the meshes can be specified a priori, or a criterion can be established that maintains the positions of the inner meshes relative to the feature. Examples of the use of some criteria are described below with the experiments in section 4 .

A mesh may be moved only when its integration domain is synchronized with the next coarser domain. At each such opportunity, the mutual positions of the innermost mesh and of the feature are compared, and if the difference between the positions in either latitude or longitude is greater than the grid spacing of the next coarser mesh, the innermost mesh is shifted by one cell of that coarser mesh. When the number of moves of the innermost mesh in one of the four (north, south, east, or west) directions becomes equal to the grid ratio between the next two meshes, the coarser mesh is subsequently shifted by one grid space of the next outer mesh, and so on. For example, in the case with triply nested meshes shown in Fig. 1, after two moves of the innermost mesh 3 in one direction, mesh 2 would be shifted in the same direction by one cell in mesh 1 . If the grids were instead in the ratio $1: 2: 6$, mesh 2 would be shifted after three moves of mesh 3. Thus, the telescopic nesting configuration is always maintained. Since during each time step a mesh can be moved by one cell of the next coarser mesh, the speed of the mesh movement is limited by the grid spacing and the time increment. However, it is not a severe restriction; by choosing a sufficiently small time step, in principle, any propagating feature can be kept within the moving mesh.

When a mesh is moved, the following must be accounted for:

1) its trailing edge becomes part of the coarser mesh and

2) at its leading edge, it takes on an area of the coarser mesh.

Because of the changes of resolution involved in conditions 1 and 2, new values need to be calculated for the affected grid points. In order to conserve mass, heat, salinity, and momentum in the course of mesh movement, a scheme developed by Kurihara et al. (1979) is employed that conserves the area integrals of these quantities over the grid cells involved.

For condition 1, a number of fine-mesh cells, $N$, are represented after the move by a single cell of the coarse mesh. In this case, the new value $F_{0}$ of a particular field at the center of a coarse-mesh cell of area $A$ is calculated by

$$
F_{0}=A^{-1} \sum_{i=1}^{N}\left(f_{i} a_{i}\right)
$$

where $f_{i}$ is the value at the center of the fine-mesh cell $i$ of area $a_{i}$. The coarse-mesh cell area $A$ is equal to the sum of the fine-mesh cell areas $a_{i}$.

For condition 2, the values represented at a single coarse-mesh point are distributed over $N$ fine-mesh cells. The new value $f_{i}$ of a fine-mesh cell of area $a_{i}$ is given by

$$
\begin{aligned}
f_{i}= & F_{0}+\left(\frac{\partial F}{c \partial \lambda}\right) c\left(\lambda_{i}-\lambda_{0}\right) \\
& +\left(\frac{\partial F}{R \partial \phi}\right)\left(\frac{A}{N a_{i}}\right) R\left(\phi_{i}-\phi_{0}\right),
\end{aligned}
$$

where $R$ is the radius of the earth; $c=R \cos \phi_{0} ;\left(\phi_{i}, \lambda_{i}\right)$ and $\left(\phi_{0}, \lambda_{0}\right)$ are the latitude and longitude positions of the fine-mesh and coarse-mesh points, respectively; and the gradients are evaluated on the coarse mesh.

It should be noted here that some dynamical imbalance between the mass and velocity fields may occur after the application of (1) and (2). As a result, highfrequency waves can be generated in the course of dynamical adjustment. However, these waves are suppressed adequately in the present model through the use of the frequency-selective time scheme and occasional spatial smoothing described above. No additional smoothing is required for the mesh movement.

\section{Test simulations}

Before examining the model performance in realistic ocean simulations, we turn in this section to results using the mesh movement algorithm under simple idealized conditions. Such tests permit comparison of the results with known solutions and establish the model's credibility for more complicated applications. A summary of all the test simulations is included in Table 1 .

\section{a. Kelvin wave propagation along the equator}

The first set of experiments simulates the propagation of an equatorial Kelvin wave, generated by the evolution of an initially imposed perturbation in the ocean thermocline. For these experiments, the model is simplified in two ways: the multilayer vertical structure is reduced to a single active upper layer (the second layer is at rest) and any vertical mixing or diffusion is neglected. The initial conditions assume zero velocity and a Gaussian bell-shaped perturbation in the upper-layer thickness, symmetric about the equator:

$$
h=H+\delta h \exp \left[-\left(x^{2}+y^{2}\right) / L^{2}\right],
$$

where $h$ is the layer thickness, $x=R\left(\lambda-\lambda_{c}\right) \cos \phi, y$ $=R\left(\phi-\phi_{c}\right), R$ is the radius of the earth, and $\lambda_{c}$ and $\phi_{c}$ are the longitude and latitude of the center of the 
TABLE 1. Summary of the test experiments.

\begin{tabular}{|c|c|c|c|c|c|}
\hline Expt & Configuration & Mesh & Mesh size & Resolution $\left({ }^{\circ}\right)$ & Time step (s) \\
\hline \multicolumn{6}{|c|}{ Kelvin wave } \\
\hline 1 & Single fine mesh & 1 & $600 \times 300$ & $2 / 15$ & 1200 \\
\hline 2 & Nested mesh & 1 & $100 \times 50$ & $4 / 5$ & 7200 \\
\hline & & 2 & $67 \times 82$ & $4 / 15$ & 2400 \\
\hline & & 3 & $72 \times 92$ & $2 / 15$ & 1200 \\
\hline \multicolumn{6}{|c|}{ Dipole } \\
\hline 3 & Single fine mesh & 1 & $600 \times 300$ & $1 / 30$ & 400 \\
\hline 4 & Nested mesh & 1 & $100 \times 50$ & $1 / 5$ & 2400 \\
\hline & & 2 & $100 \times 100$ & $1 / 30$ & 400 \\
\hline \multicolumn{6}{|c|}{ Eddy } \\
\hline 5 & Single coarse mesh & 1 & $80 \times 40$ & $1 / 2$ & 1800 \\
\hline 6 & Single fine mesh & 1 & $240 \times 120$ & $1 / 6$ & 600 \\
\hline \multirow[t]{3}{*}{7} & Nested mesh & 1 & $80 \times 40$ & $1 / 2$ & 1800 \\
\hline & & 2 & $70 \times 70$ & $1 / 6$ & 600 \\
\hline & & 3 & $64 \times 64$ & $1 / 12$ & 300 \\
\hline
\end{tabular}

perturbation, respectively. In addition, $H$ and $\delta h$ are set to $40 \mathrm{~m}$ and $60 \mathrm{~m}$, respectively, and $L=500 \mathrm{~km}$. The values assigned for the lower-layer density and the interface density difference are $1000 \mathrm{~kg} \mathrm{~m}^{-3}$ and $3 \mathrm{~kg}$ $\mathrm{m}^{-3}$, respectively, so that the long gravity wave speed $C=(g \Delta \rho H / \rho)^{1 / 2}=1.08 \mathrm{~m} \mathrm{~s}^{-1}$, and the equatorial radius of deformation $(C / 2 \beta)^{1 / 2}=153.6 \mathrm{~km}$.

To evaluate the performance of the movement scheme, we compare results using a triply nested mesh system and a benchmark integration using a single fine mesh of uniform resolution. The model domain extends from $20^{\circ} \mathrm{S}$ to $20^{\circ} \mathrm{N}$ and from $0^{\circ}$ to $80^{\circ}$ in longitude. For this experiment, and for the rest of the experiments reported in this paper, the outermost boundary of the model domain is closed with nonslip and no-flux boundary conditions. The resolution of the single mesh is the same as that of the innermost mesh of the triply nested case. The center of the perturbation (3) is initially placed in the middle of the finest mesh of the nested configuration; the initial conditions for the uniform single mesh are identical.

The outermost domain is stationary, but the inner meshes are movable and follow the eastward propagating Kelvin wave. For these experiments, the criterion for mesh movement is defined so as to keep the innermost mesh centered over the leading edge of the Kelvin wave. The leading edge of the Kelvin wave here is characterized by a sharp increase of the upper-layer thickness. It is therefore identified in this experiment as the center of mass of the region of negative zonal gradient of the thickness.

Figure 2 shows the zonal velocity fields from days 20 and 40 of the uniform fine-mesh and nested-mesh runs. The initial perturbation is dispersed into westward propagating Rossby waves and an eastward propagating Kelvin wave. Only the Kelvin wave is shown in Fig. 2. In the single-mesh run, the Kelvin wave has begun to break by day 20 , so that a sharply defined leading edge is identified. The width of the front at the leading edge is limited by the resolution, and by the degree of dissipation in the model, which here is intentionally kept low. By day 40, the gravity waves formed at the leading edge of the Kelvin wave have developed a wedgeshaped pattern, analogous in some respects to the wake of surface gravity waves behind a steadily moving ship. This is in contrast with the nested-mesh integration, in which most of the shorter wave structure is substantially reduced in the middle mesh and lost in the coarse resolution of the outermost mesh. Only in the fine-resolution mesh of the nested-mesh model do the solutions maintain the same degree of small-scale structure. This is clearly seen in Fig. 3, where we show the same fields for the domain of the nested meshes (i.e., for the inner two meshes of the nested-mesh model and for the equivalent region in the single-mesh model). The movable nested-mesh system reproduces very well the solution using the single mesh, in the region of equivalent fine resolution. In the second mesh, the solution is noticeably different because of the change of resolution. We note, however, that there is no evidence of noise generated at the mesh interfaces oriented either parallel or transverse to the direction of mesh movement.

It is appropriate to compare here the computational efficiencies of the two simulations, as the decrease in the costs associated with the moving-mesh configuration is a primary motivation for the development of this model. The uniform fine-mesh simulation requires 511 min of processor time ${ }^{1}$ and $176 \mathrm{MB}$ of core memory. In contrast, the nested-mesh run takes only $34 \mathrm{~min}$ and $25 \mathrm{MB}$ of core memory. A significant decrease in hardware requirements is achieved by the use of the movable nested-mesh model.

\footnotetext{
${ }^{1}$ The values reported here are the memory in megabytes and the single R10000 processor CPU time required on a Silicon Graphics, Inc., Origin 2000 system.
} 

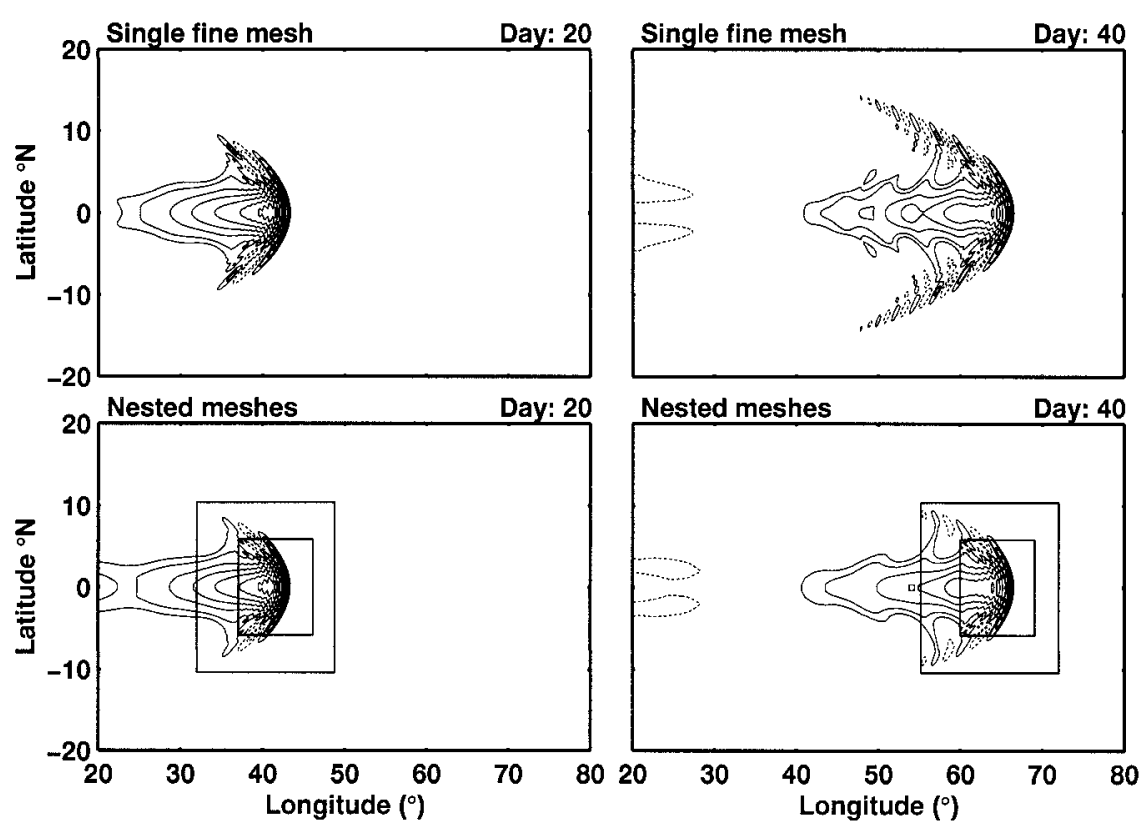

FIG. 2. The zonal velocity for the single-mesh and nested-mesh simulations of the Kelvin wave response to an initial displacement of the layer thickness field, at days 20 and 40 . The positions of the nested meshes are represented by the solid rectangles. The contour interval is $10 \mathrm{~cm} \mathrm{~s}^{-1}$; the zero contour is suppressed and the $\pm 5 \mathrm{~cm} \mathrm{~s}^{-1}$ contours are added. Negative contours are dashed. Only a portion of the computational domain is shown.

\section{b. Dipole propagation}

The next series of movable-mesh test experiments simulates the propagation of a dipolar vortex pair (a dipole). The dipole consists of two closely packed counterrotating eddies, which are capable of trapping fluid particles and transporting them over distances much larger than the initial size of the dipole. The self-induced propagation of the dipole is largely determined by its structure. Therefore, a very fine grid resolution is required to simulate it effectively. A movable-mesh system provides the ideal framework for this type of simulation. Because there is very little interaction between the dipole and its surrounding environment, one can use a very large grid ratio between the adjacent meshes; that is, it is possible to configure a model with the very fine horizontal resolution required for the dipole in an inner mesh, and with much coarser resolution in an outer mesh.

The same simplified model is applied here as in the previous section. The initial structure of the dipole is specified as a perturbation, $\delta h$, of the upper-layer thickness, $H$, as follows:

$$
\delta h=H A(r) r \sin \left(\phi-\phi_{c}\right),
$$

where $r$ and $\phi$ are polar coordinates in which $r$ is the radial distance and $\phi$ is the angle measured counterclockwise relative to eastward, $\phi_{c}$ denotes the initial angle of dipole propagation, and $A(r)$ is a nondimensional function that determines the structure of the di- pole. In the present case, $A(r)$ is defined using an analytical solution obtained by Larichev and Reznik (1976). The reader is referred to Ginis et al. (1998) for the details of the dipole initialization. In this experiment, the upper-layer thickness $H$ is set to $400 \mathrm{~m}$, and the density difference between the active and motionless layers $\Delta \rho$ is $2 \mathrm{~kg} \mathrm{~m}^{-3}$. The dipole is initialized at $35^{\circ} \mathrm{N}$. The initial velocity field is estimated from the geostrophic balance equations. For our test runs, we choose the eastward initial direction $\left(\phi_{c}=0\right)$; that is, the cyclonic vortex is to the north and the anticyclonic one is to the south.

The performance of the model configured with moving meshes is again compared with that using a single uniform mesh (Table 1). The computational domain covers the area from $30^{\circ}$ to $40^{\circ} \mathrm{N}$, and from $0^{\circ}$ to $20^{\circ}$ in longitude. For the moving-mesh system, the ratio between the grid spacing of the outer and inner meshes is set to be $6: 1$, so that 36 cells in the fine-resolution mesh correspond to one cell in the outer mesh. Movement of the inner mesh is defined to keep the dipole always within the high-resolution mesh. Here, the center of mass of the region of positive zonal velocity is used to determine the mesh movement.

Figure 4 gives an overview of the dipole movement during the course of the integration. The initial location of the dipole is $35^{\circ} \mathrm{N}$, and $3^{\circ}$ east of the western boundary. The dipole initially propagates eastward, but by day 15 of the integration has begun to propagate to the south- 

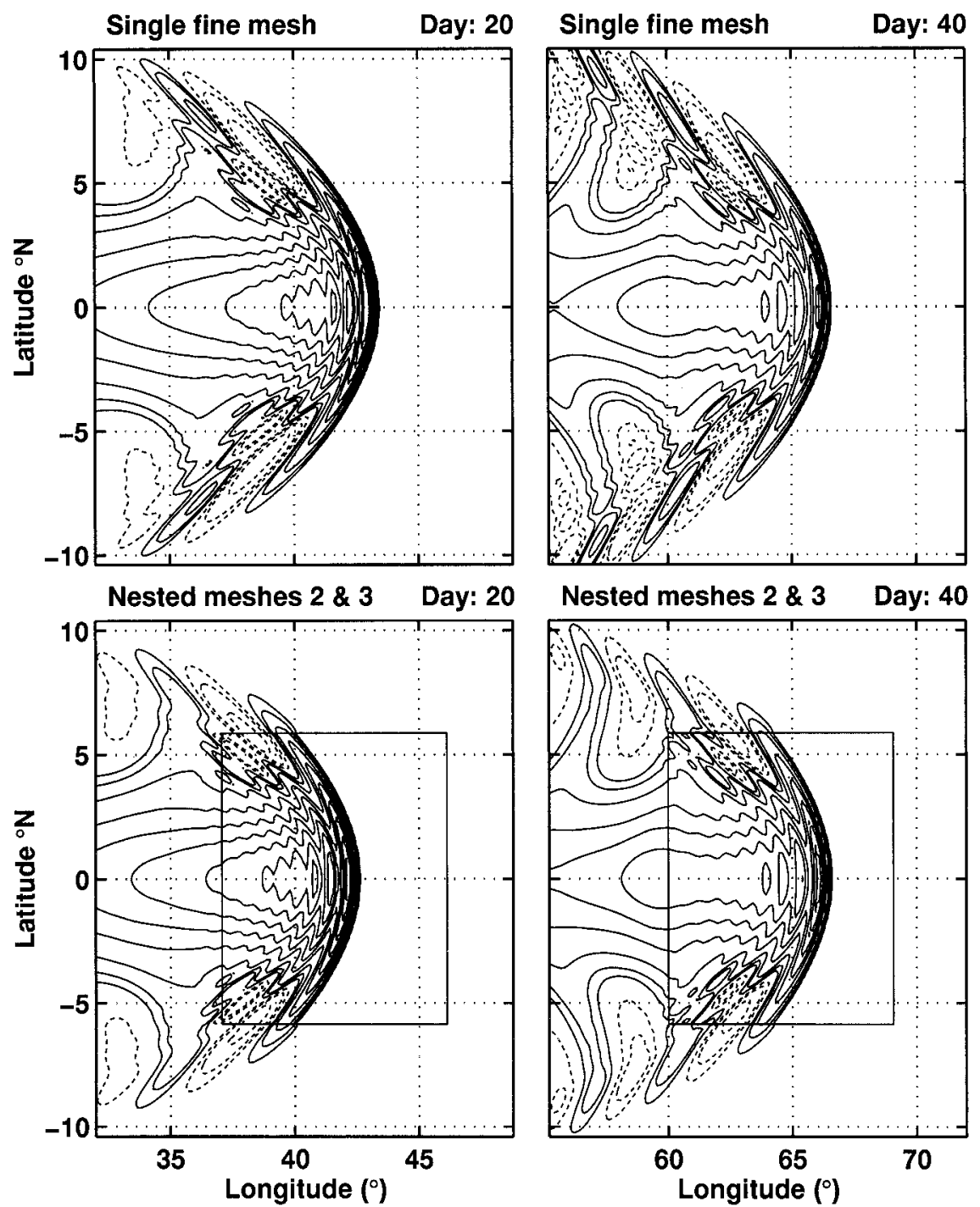

FIG. 3. As in Fig. 2 but for the inner two meshes of the nested-mesh simulation and the equivalent area of the single-mesh case. The position of the innermost nested mesh is represented by the solid rectangle. The contour interval is $10 \mathrm{~cm} \mathrm{~s}^{-1}$; the zero contour is suppressed and the $\pm 5 \mathrm{~cm}$ $\mathrm{s}^{-1}$ contours are added. Negative contours are dashed.

east. This is due to a strengthening of the southern, anticyclonic vortex relative to the cyclonic vortex to its north; this strengthening is evident in the corresponding velocity field in the figure. By day 30 , the cyclonic vortex has strengthened, due to conservation of absolute vorticity, so that the dipole reaches a minimum latitude just south of $32.5^{\circ} \mathrm{N}$ around day 50 , before propagating to the northeast. It is clearly seen that both the path and propagation speed of the dipole in the nested-mesh and single-mesh runs are very similar.

A more detailed comparison of the position and structure of the dipole for the two experiments is presented in Fig. 5. At day 75, the positions of the dipole center in the two experiments differ by $5.7 \mathrm{~km}$, and the angle of orientation by $1.5^{\circ}$. Both the small, $\sim 2 \mathrm{~cm} \mathrm{~s}^{-1}$, dif- ferences in the alongtrack velocity, and the small deviation in the eddy position and direction, demonstrate that the structure of the eddy is well maintained in the nested-mesh integration, in comparison with the finemesh results. Note that the dipole is not entirely confined in the area of the inner mesh of the nested-mesh model, as weak $\left(\sim 2 \mathrm{~cm} \mathrm{~s}^{-1}\right)$ velocities extend far enough away from the center of the dipole to reach outside the domain of the nested mesh (Fig. 5). This indicates that in the course of the moving-mesh run, some dynamical interaction may have occurred between the fine and coarse meshes during the mesh translation. However, these interactions do not appear to have caused any computational problems. The agreement between the solutions here suggests that the mesh movement algorithm han- 


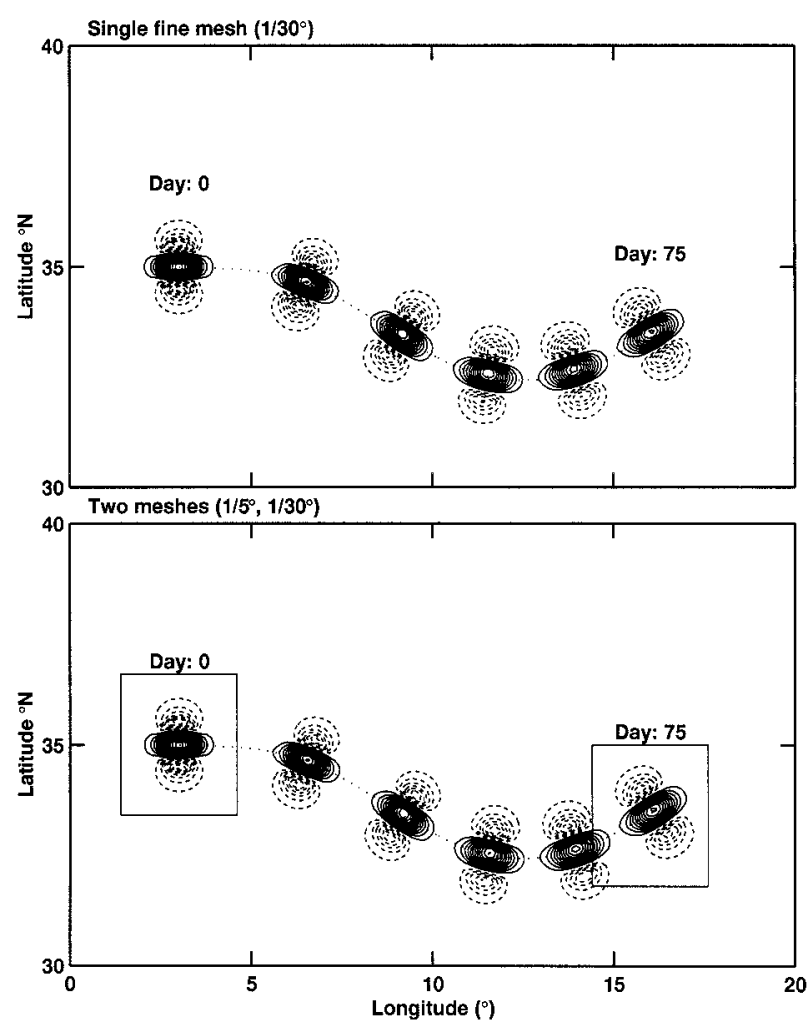

FIG. 4. The alongtrack velocity for the single-mesh and nestedmesh dipole simulations. The velocity contours for days $0,15,30$, 45, 60, and 75 are overlaid. For each time, the alongtrack (crosstrack) direction was defined normal to (along) the line between the minimum and maximum layer thicknesses. The dotted line in each plot is the track of the nested mesh for the moving-mesh simulation. The rectangles show the positions of the fine-grid mesh in the nestedmesh model at days 0 and 75 . The contour interval is $10 \mathrm{~cm} \mathrm{~s}^{-1}$, and the zero contour is suppressed.

dles these situations well, even when information is transferred from one coarse cell to 36 fine cells, and vice versa.

Remarking again on the computational efficiencies of the two configurations, the uniform fine-mesh simulation required $2358 \mathrm{~min}$ of processor time and $176 \mathrm{MB}$ of core memory, where the nested mesh run took only $122 \mathrm{~min}$ and $34 \mathrm{MB}$ of memory. The large grid ratio used for the dipole experiment makes for a significant difference in the processor time.

\section{c. Mesh movement over a mesoscale eddy}

This set of experiments addresses another important consideration: the ability of the nested meshes to move through a preexisting mesoscale feature without substantial degradation of the solution. Features that are well resolved in the coarse mesh will also be represented well in the fine mesh. The converse, though, is not true. Therefore, in designing a movable-mesh system it is important to investigate what happens to features as they are transferred between meshes on which they may or may not be well resolved.

A mesoscale disturbance that is dynamically balanced at one horizontal resolution will become unbalanced after it is transferred to a mesh with different horizontal resolution. This is because the truncation error in the finite difference form of the model equations is a function of the horizontal grid spacing. It is important that the gravity waves generated as the fields adjust to the new balanced state do not contaminate the solution near the mesh interfaces. In the two cases presented here, we examine the effects of changing resolution, both when the feature is well resolved in the coarse mesh and when it is only poorly resolved. The latter case is expected to be more demanding; that is, the change in resolution should have a greater impact on the resulting solution when a feature is only marginally resolved in the coarse mesh.

For these experiments, a single-layer configuration of the model is initialized with a Gaussian bell-shaped positive anomaly in the layer thickness. The initialization is similar to the Kelvin wave experiment described above, but now at midlatitude, so that here we simulate an anticyclonic eddy. The geostrophic relation is used to initialize the velocity field due to the thickness perturbation. The layer thickness is given by (3), but with $H$ and $\delta h$ set to $400 \mathrm{~m}$ and $200 \mathrm{~m}$, respectively. The background density and active layer density anomaly are $1000 \mathrm{~kg} \mathrm{~m}^{-3}$ and $4 \mathrm{~kg} \mathrm{~m}^{-3}$, respectively, so that the long gravity wave speed $C=(g \Delta \rho H / \rho)^{1 / 2}$ is about $4 \mathrm{~m}$ $\mathrm{s}^{-1}$. The radius of deformation $R_{\mathrm{d}}=C / f_{0}$, with $f_{0}$ at the center of the eddy $\left(25^{\circ} \mathrm{N}\right)$, is $64 \mathrm{~km}$. Two sets of experiments were performed, with $L=250 \mathrm{~km}$ (big eddy, well resolved at $1 / 2$ resolution) and with $L=100$ $\mathrm{km}$ (small eddy, only marginally resolved at $1 / 2^{\circ}$ ).

To evaluate the experiments, we compare the results using a triply nested mesh system with two integrations using single meshes of uniform coarse and fine resolution (Table 1). The domain extends from $15^{\circ}$ to $35^{\circ} \mathrm{N}$, and from $0^{\circ}$ to $40^{\circ}$ in the longitudinal direction. The resolution of the single coarse mesh is everywhere the same as that of the first, outermost mesh of the nested case. The single fine-mesh case uses the resolution of the second, middle mesh. Three different configurations are used here so that we can distinguish between the effects of mesh resolution and the effects of mesh movement and changing resolution. Figure 6 gives an overview of the eddy placement and the mesh movement during the course of the 6-day integration. The center of the nested meshes is moved at a uniform speed of 4 $\mathrm{m} \mathrm{s}^{-1}$ westward along $25^{\circ} \mathrm{N}$. The initial location of the eddy is $25^{\circ} \mathrm{N}$, and $20^{\circ}$ in longitude. In both the singleand nested-mesh cases, no additional forcing is imposed. The values of $\beta$ used for the time-integration scheme are set to 0.506 in the coarse, $1 / 2{ }^{\circ}$ resolution mesh, and 2.5 in the $16^{\circ}$ and $1 / 12$ resolution meshes.

For the experiments using the well-resolved anticyclone $(L=250 \mathrm{~km})$, the eddy does not undergo any 
a) Uniform fine mesh
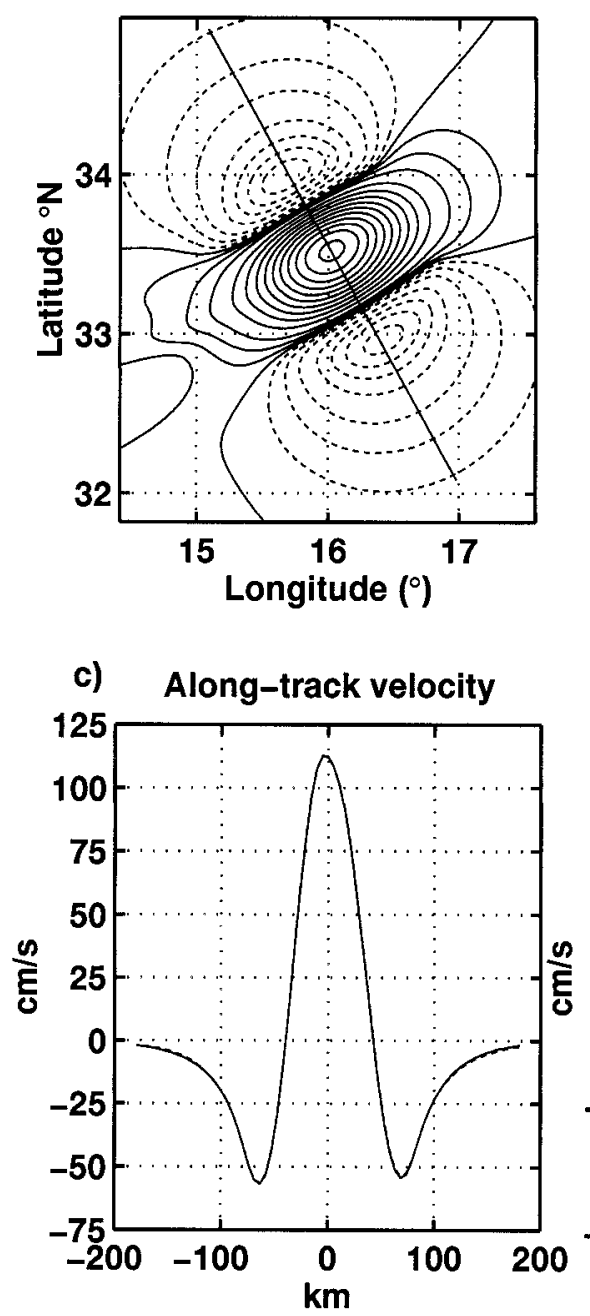

b) Nested mesh 2

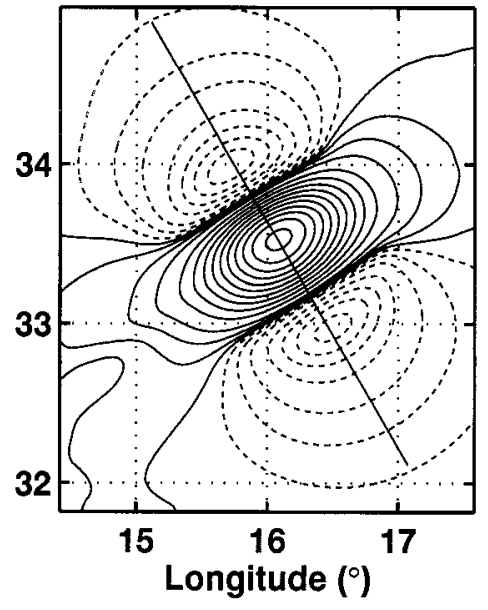

d) Velocity difference

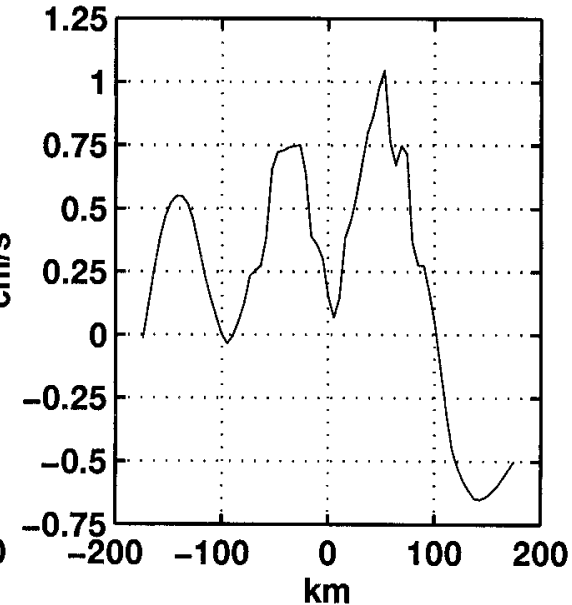

FIG. 5. The alongtrack velocity for day 75 for the uniform fine-mesh and nested-mesh dipole simulations (a), (b). The contour interval is $10 \mathrm{~cm} \mathrm{~s}^{-1}$; the zero contour is suppressed and the \pm 2.5 and $5 \mathrm{~cm} \mathrm{~s}^{-1}$ contours added. Negative contours are dashed. The cross-track structure of the alongtrack velocity through the center of the eddy [(c) uniform mesh solid, nested mesh dashed] and the difference in the two solutions (d) are also shown. In each case, the velocity in the alongtrack direction was defined as for Fig. 4, and the center of the eddy taken as the position of the apparent center of mass of the region of positive alongtrack velocity. The straight solid lines in (a) and (b) show the lines along which the velocities were compared.

substantial evolution during the integration in any of the experiments. Comparisons of the layer thickness and velocity fields in the region of the eddy reveal only slight differences in the solutions using the three mesh configurations (Fig. 7). The passage of the inner meshes through the eddy does not generate any substantial modification in its structure in this case, indicating that the geostrophic balance is maintained extremely well despite the large changes in horizontal resolution.

Plots of the meridional velocity along a zonal line through the center of the eddy give a quantitative look at the differences during and after the passage of the nested meshes (Fig. 8). At day 2, the velocities in both the fine-mesh run and the nested-mesh run indicate a slight intensification of the eddy in comparison with the coarse-mesh run. Although the eddy is well resolved in the uniform coarse mesh, the smaller grid spacing in the fine configurations still allows for steeper gradients and larger velocities to evolve. Note that the effect of the higher resolution in the nested-mesh system extends into the coarse-grid domain ahead of the mesh movement. This is a result of the two-way dynamical interaction between the adjacent meshes of differing resolution. At days 4 and 6 in the nested-mesh case, it appears that intensification of the eddy in the finer meshes, and gravity wave generation due to the change in the 

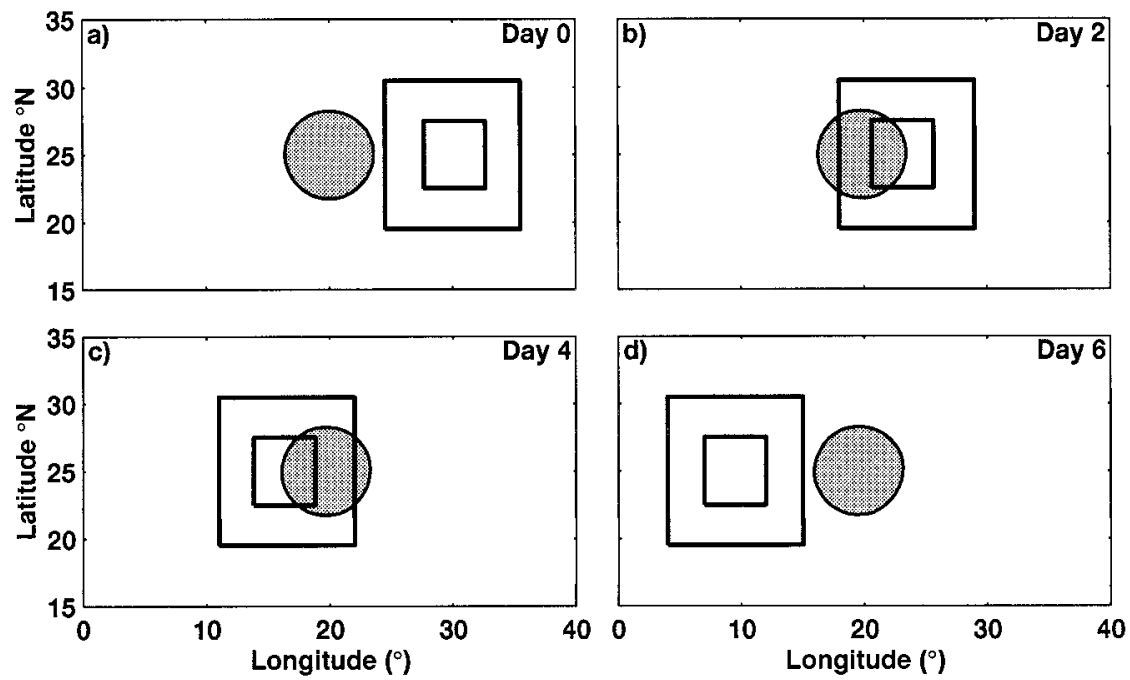

FIG. 6. (a)-(d) The positions of the nested meshes for days $0,2,4$, and 6 of a test experiment, as they move through an anticyclonic eddy. The shaded area in the center of the domain shows the position of the eddy.

grid resolution, also affect the solution in the coarsegrid mesh in the wake of the moving meshes. However, the amplitudes of the residual velocities are less than 1 $\mathrm{cm} \mathrm{s}^{-1}$ and, therefore, do not deteriorate the solution in any meaningful way. It is also important to note that no wave trapping effects are observed at the mesh interfaces because high-frequency, very short waves are effectively suppressed in the present model.

Similar comparisons of the layer thickness and velocity fields in the region of the eddy show that the change in resolution has a greater impact on the resulting solution for the poorly resolved eddy $(L=100 \mathrm{~km})$. It is apparent in Fig. 9 that the uniform coarse-mesh solution is substantially degraded, while the uniform finemesh solution maintains the structure of the small eddy (note that the area shown in Fig. 9 is smaller than that in Fig. 7). While the day 6 velocities in the coarse-grid domain of the nested-mesh model are noticeably weaker than in the fine-mesh solution, it is clear that the mesh movement did not further degrade but, rather, improved on the uniform coarse-mesh solution. It is important to note that in spite of the change in the grid structure in the nested meshes, we see no noise in the fields that we can attribute to the mesh movement, even after the passage of the nested meshes. Plots of the meridional velocity along $25^{\circ} \mathrm{N}$ give a quantitative look at the differences (Fig. 10). Over the course of the integration, the coarse-mesh solution is strongly damped in comparison with the fine-mesh fields, so there is a substantial difference between the velocities that increases with time. The nested-mesh solution shows the tendency of the fine resolution to strengthen the eddy, so that now there is a clear improvement of the solution in the wake of the moving meshes.

In summary, the results presented in this section demonstrate the ability of the mesh movement algorithm to maintain an accurate solution while tracking a moving mesoscale feature, or propagating across an existing one. However, these test experiments were performed with a simplified one-layer model without including the effects of stratification and vertical mixing processes. With additional physics and vertical modes in the full model, the noise level can be expected to be higher than it was in these one-layer cases. The performance of the mesh movement method has to be examined by applying it to the full model. Such experiments are presented in the following section.

\section{Ocean response to tropical cyclone forcing}

We turn in this section to results using the mesh movement algorithm in the full primitive equation model configuration. These experiments further demonstrate the abilities of the mesh movement technique in realistic ocean environments. They also illustrate one intended application of the model: simulation of the ocean response to tropical cyclones.

\section{a. Idealized case}

We consider first a simulation of tropical cyclone forcing applied over a resting, horizontally uniform ocean. The model is configured using the full stratified version of the model as described in Ginis et al. (1998). Since vertical turbulent mixing is a primary cause for sea surface cooling during a hurricane, adequate surface temperature prediction requires high resolution in the vertical. The number of vertical layers over the simulated upper ocean of $400 \mathrm{~m}$ is set to 11 , with higher resolution immediately below the mixed layer (Table 3 ). This latter feature enables the entrainment process to be represented with greater accuracy. 

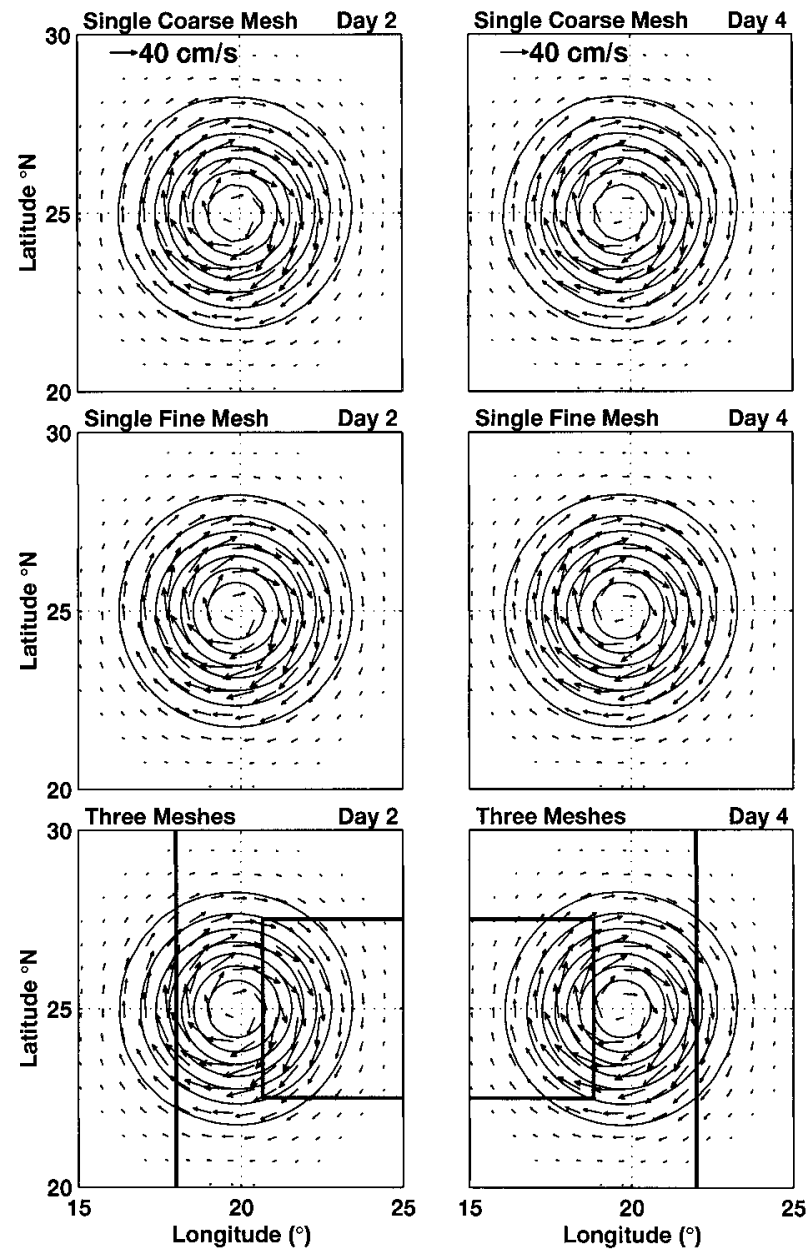

FIG. 7. Velocity vectors plotted over layer thickness contours for days 2 and 4 , for the single coarse mesh $\left.(1 / 2)^{\circ}\right)$, single fine mesh $\left(1 / 6^{\circ}\right)$, and triply nested $\left(1 / 2^{\circ}, 1 / 6^{\circ}\right.$, and $\left.1 / 12^{\circ}\right)$ solutions for the well-resolved anticyclone $(L=250 \mathrm{~km})$. The contour interval is $25 \mathrm{~m}$, and the outer closed contour $425 \mathrm{~m}$. The reference velocity vectors are plotted in the upper panels. The heavy dark lines in the lower panels show the boundaries of meshes 2 and 3 for the nested-mesh case (refer to Figs. $6 \mathrm{~b}$ and $6 \mathrm{c}$ ).

The ocean is assumed to be initially at rest and horizontally uniform. The vertical structure is defined using climatological temperature and salinity profiles in the Gulf of Mexico $\left(25^{\circ} \mathrm{N}, 90^{\circ} \mathrm{W}\right)$, taken from the August monthly averaged Generalized Digital Environmental Model ocean climatology from the Naval Oceanographic Office. The values from the climatology were first interpolated to a uniform 5-m interval. The initial mixed layer depth was then defined by the thickness of the layer with potential density within $0.4 \mathrm{~kg} \mathrm{~m}^{-3}$ of the surface value $(32.5 \mathrm{~m})$. The temperature and salinity values from the levels in this layer were averaged, and the averages were used to specify the surface temperature and salinity.

The model domain extends from $15^{\circ}$ to $35^{\circ} \mathrm{N}$, and from $0^{\circ}$ to $40^{\circ}$ in the longitudinal direction. The results using a triply nested mesh system are compared with two integrations using single meshes of uniform coarse and fine resolution (Table 2). The nested-mesh structure is configured after the GFDL hurricane prediction system referred to in the introduction and uses three nested meshes of $1^{\circ}, 13^{\circ}$, and $16^{\circ}$ resolution. The outermost domain is stationary during the integration, but the two inner meshes are movable and follow the tropical cyclone center. In the experiments with single-mesh configurations, the coarse mesh is the same resolution as the outermost nested mesh $\left(1^{\circ}\right)$, but the grid spacing of the single fine mesh matches that of the finest nested mesh $\left(1 /{ }^{\circ}\right)$.

The tropical cyclone forcing is applied as an azimuthally symmetric wind stress pattern. For the wind stress radial profile we use the linear formula of Chang and Anthes (1978),

$$
\left(\tau^{\phi}, \tau^{r}\right)=\left(\tau_{\max }^{\phi}, \tau_{\max }^{r}\right) \begin{cases}r / r_{m}, & 0<r<r_{m} \\ \left(r_{\mathrm{o}}-r\right) /\left(r_{\mathrm{o}}-r_{m}\right), & r_{m}<r<r_{\mathrm{o}}\end{cases}
$$

where $\left(\tau_{\max }^{\phi}, \tau_{\max }^{r}\right)=(3.0 \mathrm{~Pa},-1.0 \mathrm{~Pa})$ are the maximum azimuthal and radial components of the wind stress, $r$ is the radial distance, and $r_{m}=50 \mathrm{~km}$ and $r_{\mathrm{o}}=300$ $\mathrm{km}$ are the radii of the maximum wind and the outer edge of the cyclone, respectively. The cyclone center is moved westward along $25^{\circ} \mathrm{N}$ at a speed of $5 \mathrm{~m} \mathrm{~s}^{-1}$.

Figure 11 shows the sea surface temperature and velocity fields for part of the model domain at day 4 of the integrations. The cold temperatures and strong nearinertial oscillations in the wake of the storm passage are well developed at this time, when the center of the storm is located near $13^{\circ}$ longitude. In the fine mesh, the maximum cooling in the storm wake is near $3.7^{\circ} \mathrm{C}$.

Attention should be paid to several features of this comparison. First, we notice that both the temperature and velocity signals in the wake are substantially weaker in the uniform coarse-mesh solution than in the finemesh solution. For the velocity field, this is to be expected as the coarse mesh is not sufficiently fine to resolve the tropical cyclone wind structure, since the grid spacing is more than twice as large as the radius of maximum wind. In addition, the near-surface velocities decay more rapidly behind the storm as the low horizontal resolution cannot sustain large horizontal velocity shears. Because vertical mixing is the primary mechanism for the SST decreases, the weaker currents in the coarse-mesh case generate much less surface cooling in the storm's cold wake.

The solution in the finest mesh of the nested-mesh solution reproduces very well the uniform fine-mesh solution. Even in the middle mesh, only slight differences in the fields are noticeable. It is also seen that the nested-mesh model has even improved the SST solution in the coarsest mesh. Although the velocities in the wake are comparable with those in the uniform coarse-mesh solution, the stronger surface cooling produced under 

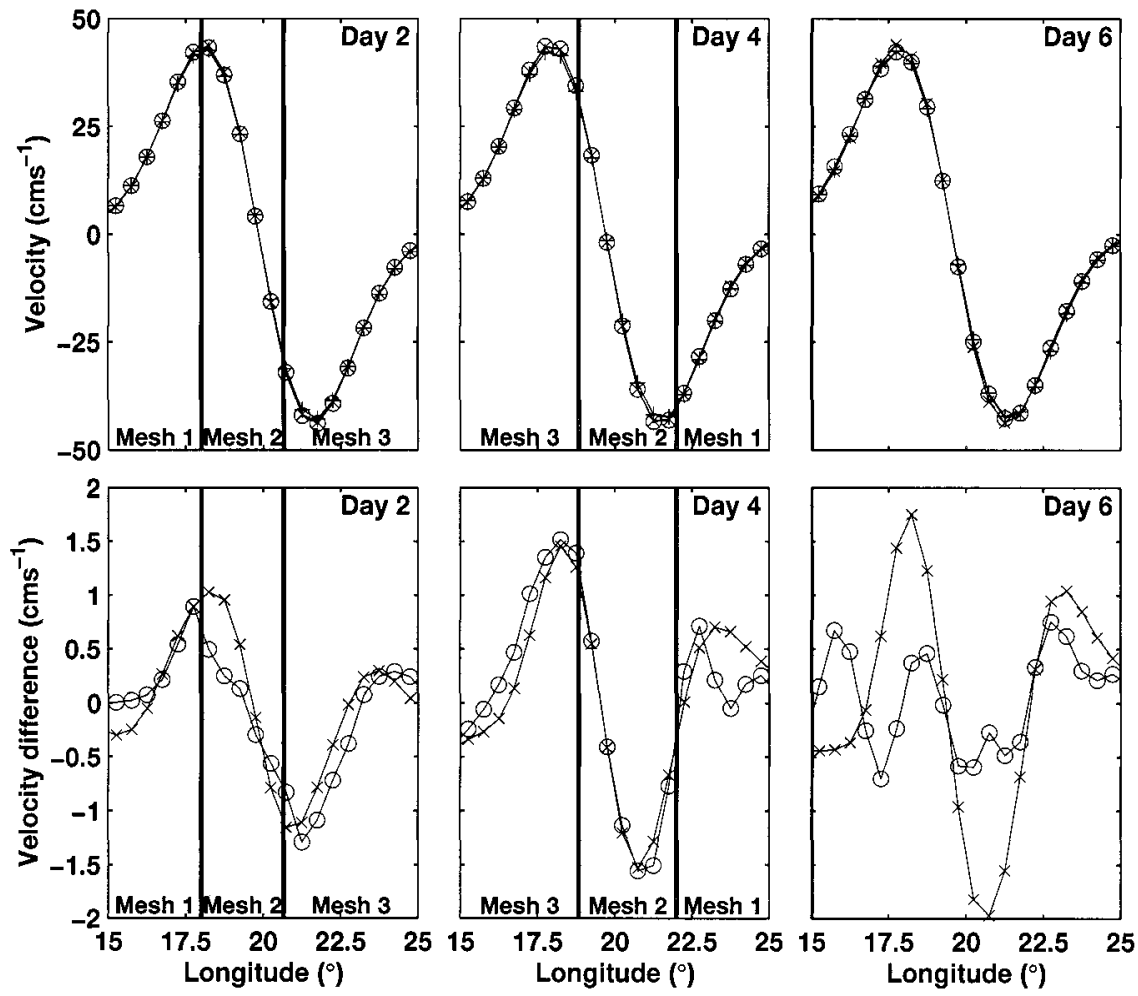

FIG. 8. (upper panels) The meridional velocity along $25^{\circ} \mathrm{N}$ for days 2,4 , and 6 for the wellresolved eddy $(L=250 \mathrm{~km})$. The dark lines show the boundaries of meshes 2 and 3 for the nestedmesh case (refer to Figs. 6b-d). The single coarse mesh $(+)$, single fine mesh $(\times)$, and triply nested $(\bigcirc)$ solutions are plotted at the coarse-mesh grid points. (lower panels) The difference from the uniform coarse-mesh solution in the meridional velocity for the same days, for the single fine mesh $(\times)$ and triply nested $(\bigcirc)$ solutions. Note the difference in the vertical scales.

the finer resolution in the innermost mesh is largely maintained in the outermost coarse mesh.

To further quantify the nested-mesh model performance we compare the area-average SST values calculated for the uniform fine- and nested-mesh solutions at day 4 , for the area $23^{\circ}$ to $27^{\circ} \mathrm{N}$, and $11^{\circ}$ to $19^{\circ}$ in longitude. This area extends from about $2^{\circ}$ ahead of to $6^{\circ}$ behind the storm center, which was near $13^{\circ}$ longitude at this time, and to $2^{\circ}$ north and south of the track (refer to Fig. 11). Note that in the nested-mesh case, this area extends into the second mesh. The uniform fine-mesh and nested-mesh values of $28.25^{\circ}$ and $28.24^{\circ} \mathrm{C}$ compare very well. The moving-mesh system appears to give a proper SST solution in the vicinity of the storm forcing. The implication of this result is quite important. Accurate surface temperature predictions are key to tropical cyclone-ocean interactions because of the central importance of surface heat fluxes to cyclone intensity.

In Fig. 12 we compare the vertical structure of the temperature and meridional velocity in the wake of the cyclone for the three cases. This zonal slice is taken just to the right of the storm track, near the radius of maximum wind $\left(25.5^{\circ} \mathrm{N}\right)$. It is seen that the cyclone also generates a strong baroclinic response that includes three-dimensional, near-inertial gravity waves. Vertical motions induced by the divergence of the mixed layer currents are apparently more energetic in the uniform fine-mesh solution. Again, there is clear agreement between the uniform fine-mesh and nested-mesh solutions for the area near the storm. Outside of the second mesh in the nested-mesh model, the velocities and the amplitudes of the near-inertial gravity waves decay rapidly, similar to the coarse-mesh solution. An examination of the model fields in the nested-mesh solution reveals no distortions in the vicinity of the mesh interfaces, which are frequently the location of spurious wave disturbances in nested-mesh models with ill-posed interface conditions. Thus, the moving-mesh system does not appear to have any difficulty with the addition of vertical stratification and full thermodynamics.

These experiments demonstrate that the present nested-mesh model, with the mesh movement technique implemented, may successfully be applied to predictions of the ocean response to tropical cyclones and the associated cold wake at the sea surface.

\section{b. Real-case simulation in the western North Pacific}

In the last experiment in this paper, the mesh movement technique is applied to a simulation of the ocean 


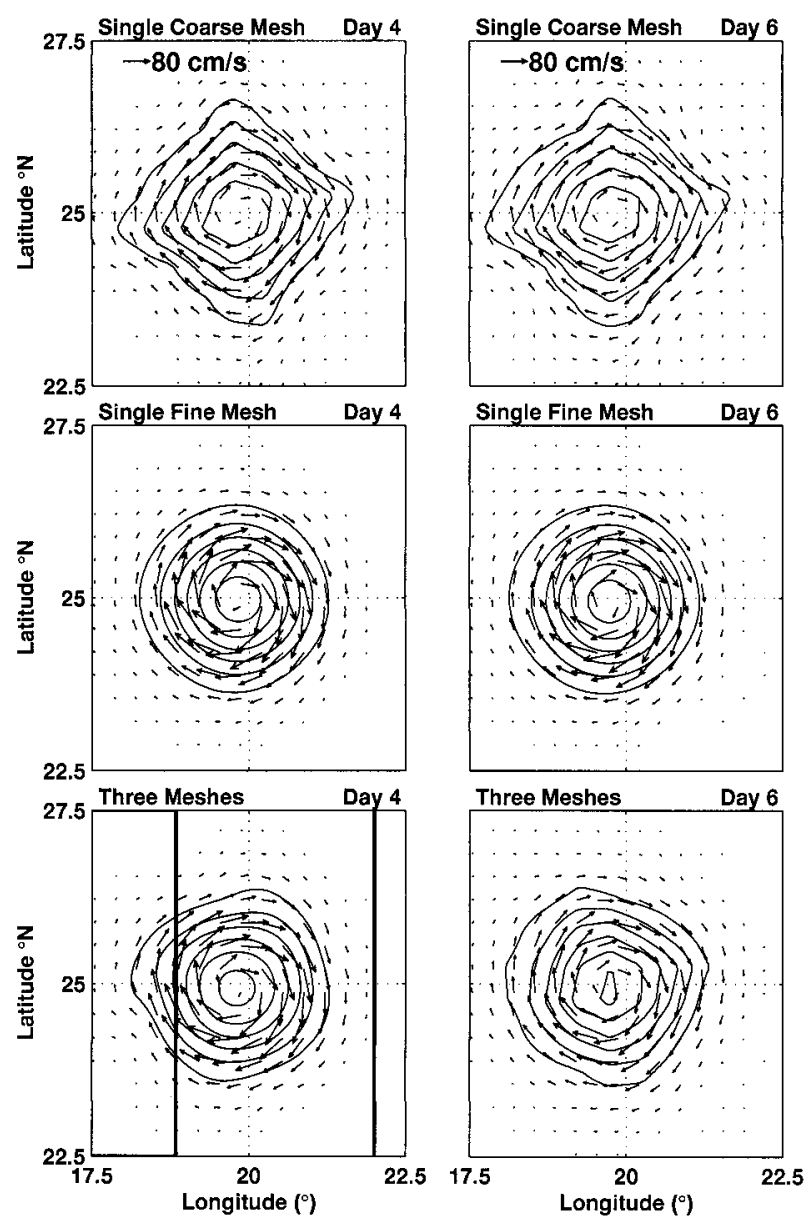

FIG. 9. As in Fig. 7 but with the horizontal scale of the eddy reduced to $L=100 \mathrm{~km}$. The fields are shown for days 4 and 6 of the smaller area. Refer to Figs. $6 \mathrm{c}$ and $6 \mathrm{~d}$ for the inner-mesh positions.

response to a real-case typhoon in the western North Pacific. The purpose of the present simulation is to establish the model's credibility in a realistic ocean environment. We envision that the application of the nested-mesh model will be particularly valuable for tropical cyclones in the Pacific Ocean, because its large size makes it computationally infeasible to use a very high resolution mesh for the entire basin.

We apply the nested-mesh model to examine the ocean response to Typhoon Roy in January 1988 (Fig. 13). Roy was a very destructive storm, although it was the first major tropical cyclone of the season in the western North Pacific. The cyclone formed over above normal sea surface temperature anomalies in the central Pacific. Roy moved about $7400 \mathrm{~km}$ in 11 days and caused significant damage on Kwajalein Atoll and the islands of Guam and Rota, before crossing Luzon in the Phillipines and dissipating over the South China Sea (Joint Typhoon Warning Center 1988).

For the present experiment, the model was initialized using the January fields from year 6 of a multiyear spinup integration. During the model spinup, a single mesh was used with $1^{\circ}$ resolution in the zonal direction and variable resolution in the meridional direction that ranged from $13^{\circ}$ near the equator to $2.5^{\circ}$ at the northern and southern boundaries. The computational domain covers the entire tropical Pacific region from $30^{\circ} \mathrm{S}$ to $30^{\circ} \mathrm{N}$ and from $124^{\circ} \mathrm{E}$ to $70^{\circ} \mathrm{W}$ with a realistic but simplified coastline. The model was initialized using the annual mean Levitus (1982) temperature and salinity in a nine-layer configuration. The mixed layer depth was determined according to a vertical gradient of density criterion, and the $1027 \mathrm{~kg} \mathrm{~m}^{-3}$ density level was specified as the base of the thermocline. The thermocline region was divided into eight layers according to the specified sigma coordinate values (Table 3 ). The model was started from rest and forced with monthly climatological surface stress and heat fluxes. The temperature and salinity values near the lateral boundaries to the north and south (poleward of $25^{\circ} \mathrm{N} / \mathrm{S}$ ) were gradually relaxed toward the climatology. The spinup simulation was integrated for $6 \mathrm{yr}$, which was sufficient to establish the quasi-equilibrium state used to initialize the present tropical cyclone experiment.

Time series of the typhoon position, maximum wind speed, and minimum surface pressure provided in the Joint Typhoon Warning Center (Guam) typhoon analysis archive (Joint Typhoon Warning Center 1998) were used to define the forcing for the tropical cyclone simulation. The radial profile of the tangential component of the wind is based on the model of axisymmetric wind circulation in a hurricane proposed by Holland (1980). The radial component is calculated from an empirical relation between radius and inflow angle (Price et al. 1994), and asymmetry in the wind field is introduced by adding half the storm translation speed (NOAA/National Weather Service 1979). The wind stress for wind speeds less than $35 \mathrm{~m} \mathrm{~s}^{-1}$ is calculated by the usual bulk formula using the drag coefficient as suggested by Large and Pond (1981). For higher wind speeds, the wind stress is reduced from the Large and Pond (1981) value to account for the increased effect of wave breaking on the surface momentum flux. This empirical wind model was successfully tested for the ocean response to Hurricane Gilbert (1988) using airborne field observations (Ginis et al. 1996).

For the tropical cyclone simulation, we introduced a triply nested configuration, in which the nested meshes of higher resolution track the center of the storm. The outermost mesh covers the same domain as in the spinup run, but with a uniform $1^{\circ}$ resolution. The parameters of the nested-mesh system are listed in Table 2. The tropical cyclone forcing was applied for the period 0000 UTC 8 January 1988 to 0000 UTC 15 January 1988. The initial positions of the two inner meshes were centered on the location of Typhoon Roy at 0000 UTC 8 January.

The surface temperature field for the entire domain for 0000 UTC 14 January is plotted in Fig. 14, which also shows the typhoon track and the relative sizes of 

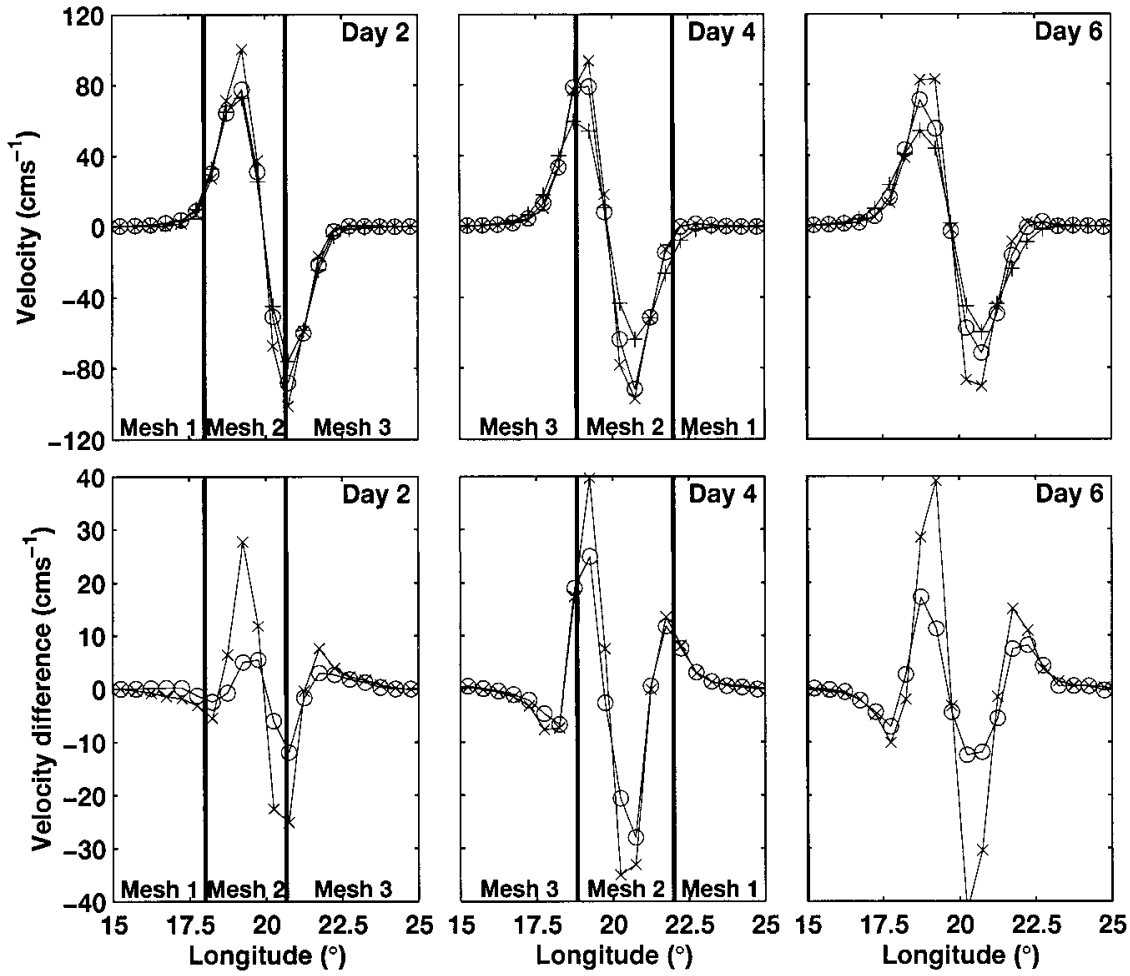

FIG. 10. As in Fig. 8 but with the horizontal scale of the eddy reduced to $L=100 \mathrm{~km}$. Refer to Figs. $6 \mathrm{~b}-\mathrm{d}$ for the inner-mesh positions.

the fixed outer mesh and the moving nested meshes. Typhoon Roy tracked the edge of the equatorial Pacific warm pool region, in which surface temperatures are greater than about $28^{\circ} \mathrm{C}$. The cold wake produced by the storm is just noticeable in this figure, but it is evident that the mesh movement introduced no distortions in the background temperature field. This demonstrates the ability of the mesh movement algorithm to maintain an accurate solution in the presence of both strong mechanical forcing and the existing large-scale circulation.

Figure 15 shows the surface temperature and velocity fields in the vicinity of the storm. On this scale, the cold wake is more easily distinguished as the region of cooler surface temperatures to the right of the storm track.

TABLE 2. Summary of the tropical cyclone experiments.

\begin{tabular}{|c|c|c|c|c|c|}
\hline Expt & Configuration & Mesh & Mesh size & $\begin{array}{l}\text { Resolution } \\
\left({ }^{\circ}\right)\end{array}$ & $\begin{array}{l}\text { Time } \\
\text { step (s) }\end{array}$ \\
\hline \multicolumn{6}{|c|}{ Idealized case } \\
\hline 1 & Single coarse mesh & 1 & $40 \times 20$ & 1 & 1800 \\
\hline 2 & Single fine mesh & 1 & $240 \times 120$ & $1 / 6$ & 300 \\
\hline \multirow[t]{4}{*}{3} & Nested mesh & 1 & $40 \times 20$ & 1 & 1800 \\
\hline & & 2 & $33 \times 33$ & $1 / 3$ & 600 \\
\hline & & 3 & $30 \times 30$ & $1 / 6$ & 300 \\
\hline & \multicolumn{5}{|c|}{ Typhoon Roy } \\
\hline 4 & Nested mesh & 1 & $166 \times 60$ & 1 & 1800 \\
\hline & & 2 & $33 \times 33$ & $1 / 3$ & 600 \\
\hline & & 3 & $30 \times 30$ & $1 / 6$ & 300 \\
\hline
\end{tabular}

Several interesting features of the wake should be noted. With reference to Fig. 13, we see that the greatest cooling produced by the storm took place after the storm reached its peak intensity. We can suggest two possible explanations for this. The first is the change in the storm translation speed, which fell from about $11 \mathrm{~m} \mathrm{~s}^{-1}(21$ $\mathrm{kt})$, from 10 to 11 January, to less than $6 \mathrm{~m} \mathrm{~s}^{-1}(11 \mathrm{kt})$, from 12 to 13 January. The second relates to the upperocean heat content below the storm. When Roy was near maximum intensity, it was still moving over the warm pool region, where the ocean near-surface heat content was much greater. Only when the storm moved away from the warm pool did it begin to produce significant (more than $2^{\circ} \mathrm{C}$ ) surface cooling.

Roy also excited very energetic surface currents and near-inertial gravity waves. A careful analysis of the results indicates that the impact of the mesh movement on the currents and wave propagation is minimal. We wish to emphasize here not only the importance of the increased model resolution in the nested-mesh model for better determining the characteristics of the ocean response to the strong atmospheric forcing, but also for better representing the large-scale flow and the nonlinear interactions of the storm-induced currents with the background circulation.

\section{Summary and remarks}

The mesh movement scheme implemented in the multiply nested primitive equation ocean model of Ginis et 

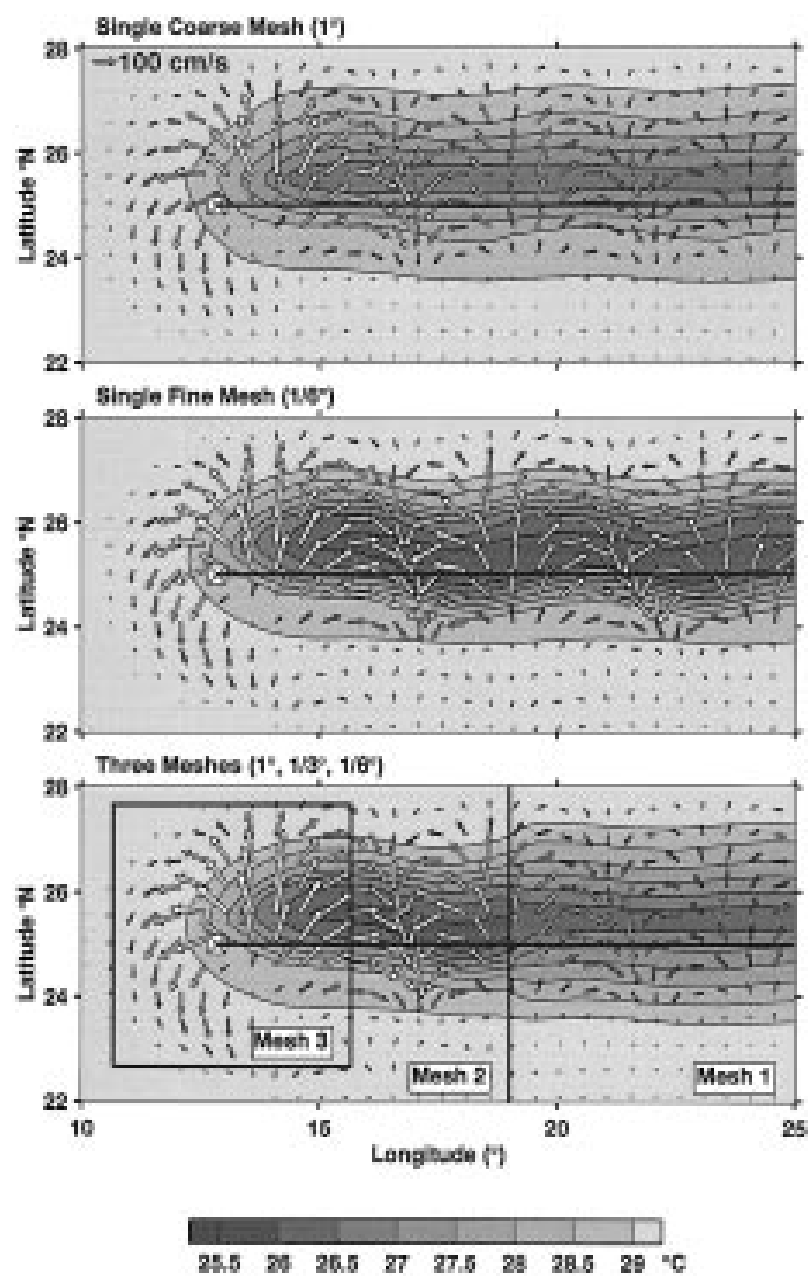

FIG. 11. Velocity vectors plotted over the surface temperature $\left({ }^{\circ} \mathrm{C}\right)$ for day 4 , for the single coarse mesh $\left(1^{\circ}\right)$, single fine mesh $\left(1 / 6^{\circ}\right)$, and triply nested $\left(1^{\circ}, 13^{\circ}\right.$, and $\left.1 / 6^{\circ}\right)$ solutions for the response to idealized tropical cyclone forcing. The shaded contours are at a $0.5^{\circ} \mathrm{C}$ interval, and the reference velocity vector is plotted in the upper panel. The storm track is shown as the solid horizontal line, and the storm position is indicated with the " $\times$." The dark lines in the lower panel also show the boundaries of meshes 2 and 3 for the nested-mesh case. Only a portion of the computational domain is shown.

al. (1998) has been described, along with idealized and realistic experiments that demonstrate its use. The use of movable nested meshes allows for the efficient application of high horizontal resolution to the simulation of features that may propagate over large distances. The model employs the technique originally proposed in $\mathrm{Ku}-$ rihara et al. (1979) and successfully applied for many years in the NOAA Geophysical Fluid Dynamics Laboratory hurricane prediction model (Kurihara et al. 1998).

Mesh movement can be specified a priori, or determined in the course of the model run, so as to follow an evolving ocean feature, such as a wave front or propagating eddy, or atmospheric forcing, such as a tropical cyclone. The nested meshes may be placed anywhere
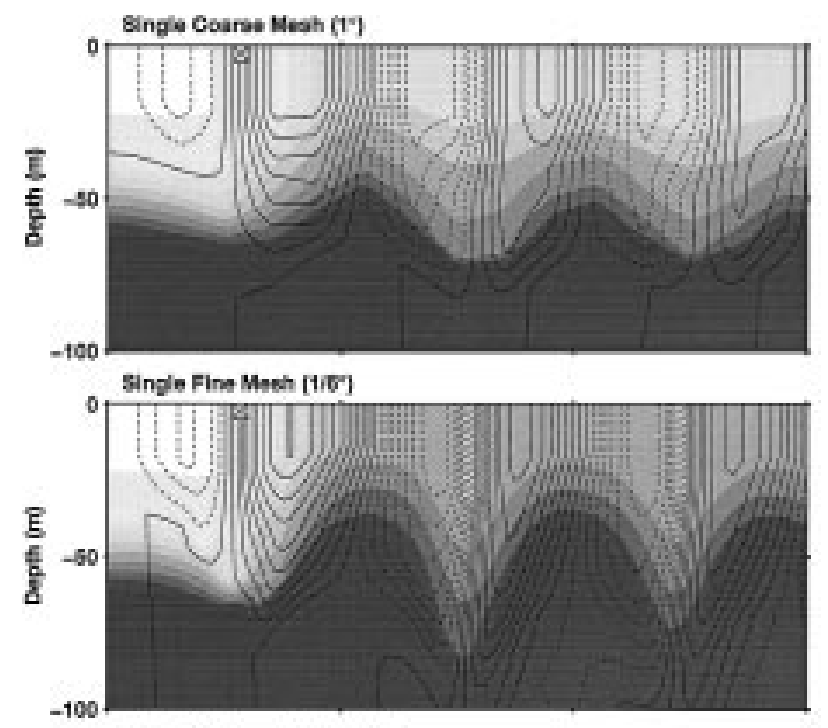

Throw Moshes (1:, 1/8, 1/8)
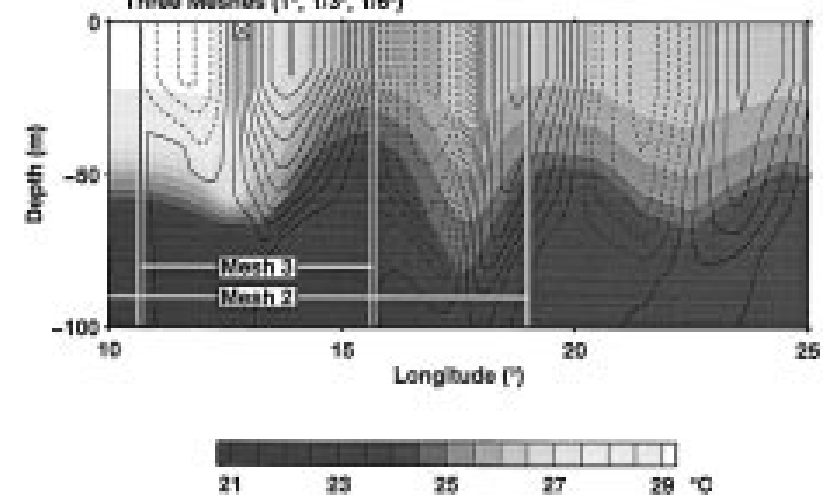

FIG. 12. The temperature and meridional velocity along $25.5^{\circ} \mathrm{N}$ at day 4 for the single coarse mesh $\left(1^{\circ}\right)$, single fine mesh $\left(1 / 6^{\circ}\right)$, and triply nested $\left(1^{\circ}, 1 / 3^{\circ}\right.$, and $1 / 6$ ) solutions for the response to idealized tropical cyclone forcing. The temperature is shaded and the velocity is contoured. The temperature shading is scaled to emphasize the temperature gradients below the mixed layer. The velocity contour interval is $20 \mathrm{~cm} \mathrm{~s}^{-1}$, and negative (southward) velocities are dashed. The heavy dark lines in the lower panel show the boundaries of meshes 2 and 3 for the nested-mesh case. Only a portion of the full computational domain is shown.

within the model domain. Mesh movement speed is limited by the grid spacing and time step, but by choosing a small time step, any propagating feature, in principle, can be tracked with the moving meshes. The direction of mesh movement is not constrained except by the outermost boundary of the model domain. Resolution changes at the leading and trailing edges of a moving mesh require that new values be determined by interpolation. The method proposed by Kurihara et al. (1979) is used so that mass, heat, and momentum are conserved during the movement.

Nonlinear computational instability in this model is effectively suppressed by the use of a frequency-selective time-integration method of Kurihara and Tripoli (1976), and by the occasional application of a spatial 


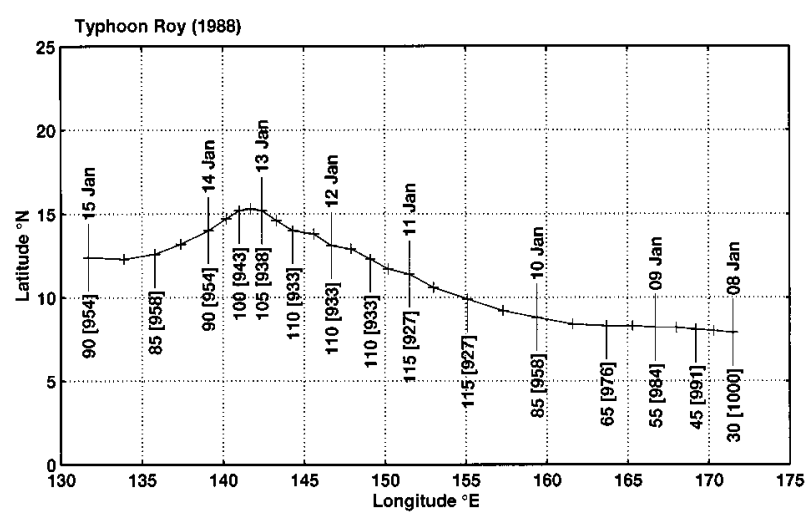

FIG. 13. The track of Typhoon Roy (1988), with the maximum wind and minimum pressure. Positions each $6 \mathrm{~h}$ are marked with "+'s," dates are labeled at the 0000 UTC position, and maximum wind speed $(\mathrm{kt})$ and minimum surface pressure $(\mathrm{hPa})$ values are noted every $12 \mathrm{~h}$.

smoothing-desmoothing technique described in Bender et al. (1993b). No additional smoothing is required with the addition of the mesh movement to the fixed-mesh model.

We described idealized and realistic tests of the movement of nested meshes in one-layer and vertically stratified versions of the model, either following a propagating feature (a dipole or an equatorial Kelvin wave, tropical cyclone forcing), moving across an existing mesoscale feature (an anticyclonic eddy), or both (the ocean response to Typhoon Roy). The results of these test cases demonstrate that the mesh movement technique works very well; that is, grid movement in the course of the integration does not distort the results, nor does it degrade the solution by generating computational noise near the mesh boundaries.

The equatorial Kelvin wave experiment showed that the model maintains an accurate fine-grid solution in the innermost moving mesh, when the results are com-
TABLE 3. The sigma-layer thickness values and nominal layer thickness values for the experiments with tropical cyclone forcing. For the idealized case, the layer thickness distribution here is an example where the full depth of the simulated upper ocean is $400 \mathrm{~m}$ and the mixed layer thickness is $32.5 \mathrm{~m}$. For the Typhoon Roy case, the layer thickness is an example where the full depth of the simulated upper ocean is $410 \mathrm{~m}$ and the mixed layer thickness is $60 \mathrm{~m}$.

\begin{tabular}{|c|c|c|c|c|}
\hline \multirow[b]{2}{*}{ Layer } & \multicolumn{2}{|c|}{ Idealized case } & \multicolumn{2}{|c|}{ Typhoon Roy } \\
\hline & $\sigma$ & $\begin{array}{l}\text { Layer } \\
\text { thickness } \\
\text { (m) }\end{array}$ & $\sigma$ & $\begin{array}{l}\text { Layer } \\
\text { thickness } \\
\text { (m) }\end{array}$ \\
\hline 1 & - & 32.5 & - & 60 \\
\hline 2 & .0136 & 5 & .0286 & 10 \\
\hline 3 & .0272 & 10 & .0429 & 15 \\
\hline 4 & .0408 & 15 & .0714 & 25 \\
\hline 5 & .0544 & 20 & .1429 & 50 \\
\hline 6 & .0816 & 30 & .1429 & 50 \\
\hline 7 & .1088 & 40 & .1429 & 50 \\
\hline 8 & .1361 & 50 & .1429 & 50 \\
\hline 9 & .1633 & 60 & .2857 & 100 \\
\hline 10 & .1633 & 60 & & \\
\hline 11 & .2109 & 77.5 & & \\
\hline
\end{tabular}

pared with a uniform fine-mesh result. The model can maintain accurate solutions with a grid ratio as large as $6: 1$, as demonstrated by the dipole simulation, which showed that the structure of the eddy was maintained over the 75-day integration. In the tests of mesh movement over existing anticyclonic eddies, the moving meshes treated the eddies without introducing distortions or instabilities, and the improvement in the solution due to the fine resolution extended into the coarsemesh domain.

Simulations of the ocean response to tropical cyclone forcing demonstrated that the moving meshes did not degrade the solution, even with the application of the strong winds and generation of energetic surface currents and near-inertial gravity waves. In comparisons with uniform fine-mesh and coarse-mesh models, the

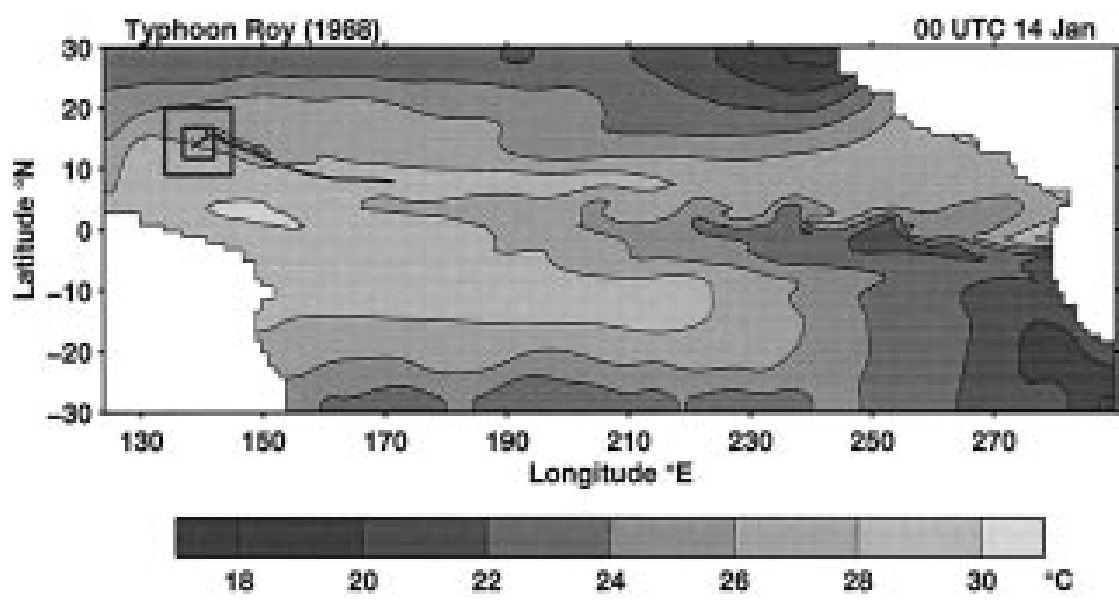

FIG. 14. The surface temperature $\left({ }^{\circ} \mathrm{C}\right)$ at 0000 UTC 14 Jan from the simulation of the ocean response to Typhoon Roy. The entire model domain is shown, plotted with the position of the nested meshes and the typhoon track. The shaded contours have a $2^{\circ} \mathrm{C}$ interval. 

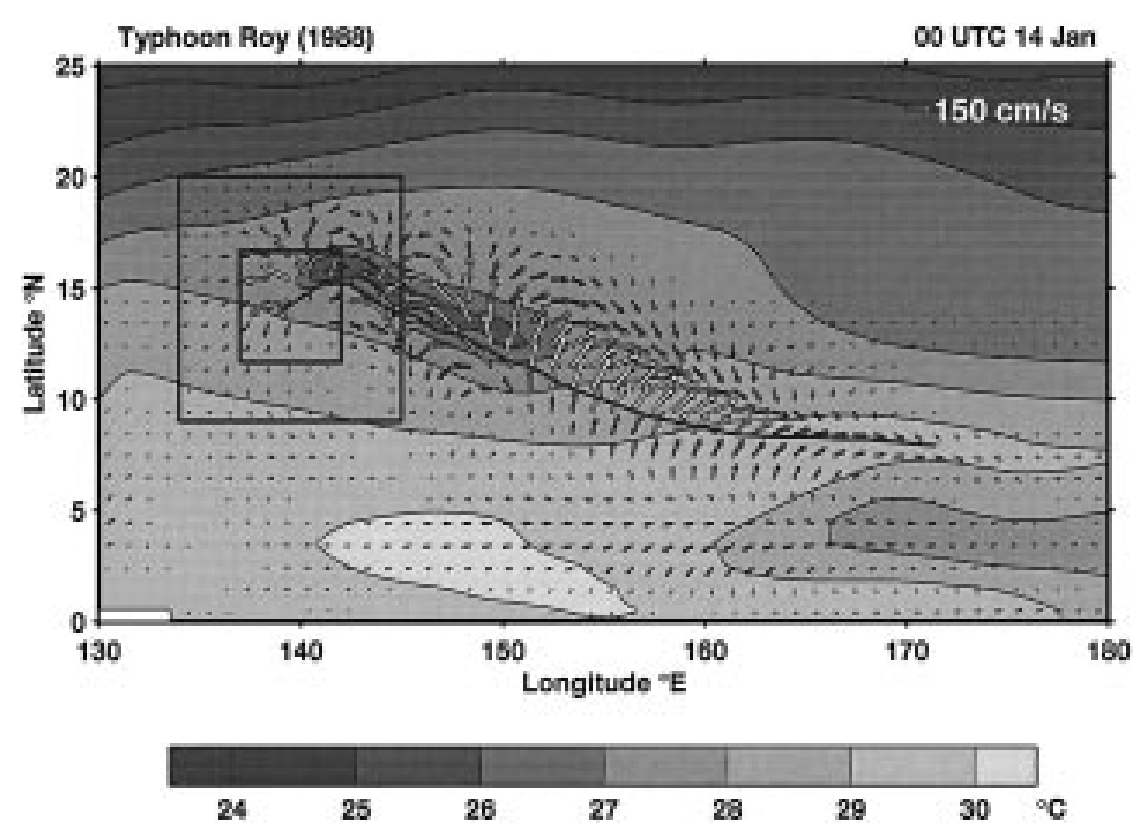

FIG. 15. Velocity vectors plotted over the surface temperature $\left({ }^{\circ} \mathrm{C}\right)$ at 0000 UTC 14 Jan from the simulation of the ocean response to Typhoon Roy. Only the area near the storm track is plotted. The shaded contours have a $1^{\circ} \mathrm{C}$ interval, and the $150 \mathrm{~cm} \mathrm{~s}^{-1}$ reference velocity vector is plotted.

moving-mesh solution reproduced the near-surface temperatures and velocities of the uniform fine-mesh solution in the inner meshes, and improved (over the uniform coarse-mesh solution) the surface temperature prediction in the cold wake, even in the coarse-mesh domain. In a simulation of the ocean response to the observed wind forcing of Typhoon Roy (1988) in the western North Pacific, the nested-mesh solution showed no difficulty in simulating the interaction of the windinduced currents with the existing background circulation. The success of the moving-mesh system in modeling the ocean response and associated cold wake at the sea surface suggests that the model may successfully be applied to predictions of the ocean response to tropical cyclones in future coupled hurricane-ocean configurations.

The model has other advanced features described in more detail in Ginis et al. (1998), including realistic coastline geometry and spatially variable grid spacing, which make it suitable for realistic simulations of the mesoscale dynamics of the sea. It is presently limited, however, by the reduced gravity assumption. A full barotropic mode over variable bottom topography, and the ability to move the nested meshes over islands and coastlines, are currently being added to the model.

Future development for this model also includes a full coupling with the GFDL tropical cyclone prediction model (Kurihara et al. 1998). The uncoupled version of the GFDL model is presently used for official operational forecasts at the National Centers for Environmental Prediction for all tropical cyclones in both the eastern Pacific and Atlantic basins. A United States Navy implementation of the GFDL model is also used at the Fleet Numerical Meteorology and Oceanography Center for tropical cyclones in the western North Pacific. The new movable-mesh ocean model described in this paper shares the same mesh nesting, time integration, and mesh movement techniques used in the GFDL hurricane model, and therefore the two models can be efficiently coupled. It is anticipated that the new coupled tropical cyclone-ocean prediction system will greatly improve forecasters' operational predictive capabilities for these dangerous storms.

Acknowledgments. This work was supported by the United States Office of Naval Research through Grant N000149610758. The authors are grateful to Y. Kurihara and M. Bender at GFDL for providing access to the GFDL hurricane model code.

\section{REFERENCES}

Bender, M. A., I. Ginis, and Y. Kurihara, 1993a: Numerical simulations of the tropical cyclone-ocean interaction with a highresolution coupled model. J. Geophys. Res., 98, 23 245-23 263.

—, R. J. Ross, R. E. Tuleya, and Y. Kurihara, 1993b: Improvements in tropical cyclone track and intensity forecasts using the GFDL initialization system. Mon. Wea. Rev., 121, 2046-2061.

Bryan, K., 1966: A scheme for numerical integration of the equations of motion on an irregular grid free of nonlinear instability. Mon. Wea. Rev., 94, 39-40.

Chang, S. W., and R. A. Anthes, 1978: Numerical simulations of the ocean's nonlinear, baroclinic response to translating hurricanes. J. Phys. Oceanogr., 8, 468-480. 
Chen, D., L. M. Rothstein, and A. J. Busalacchi, 1994: A hybrid vertical mixing scheme and its application to tropical ocean models. J. Phys. Oceanogr., 24, 2156-2179.

Falkovich, A. I., A. P. Khain, and I. Ginis, 1995: The influence of air-sea interaction on the development and motion of a tropical cyclone: Numerical experiments with a triply nested model. $M e$ teor. Atmos. Phys., 55, 167-184.

Fox, A. D., and S. J. Maskell, 1995: Two-way interactive nesting of primitive equation ocean models with topography. J. Phys. Oceanogr., 25, 2977-2996.

Gent, P. R., and M. A. Cane, 1989: A reduced gravity, primitive equation model of the upper equatorial ocean. J. Comput. Phys., 81, 444-480.

Ginis, I., 1995: Ocean response to tropical cyclone. Global Perspectives on Tropical Cyclones, R. L. Elsberry, Ed., World Meteorological Organization, 198-260.

— L. K. Shay, L. M. Rothstein, and S. A. Frolov, 1996: Numerical simulations of the ocean response to Hurricane Gilbert using airborne field observations. Preprints, Eight Conf. on Air-Sea Interaction, Atlanta, GA, Amer. Meteor. Soc., 33-37.

- - R. Richardson, and L. M. Rothstein, 1998: Design of a multiply nested primitive equation ocean model. Mon. Wea. Rev., 126, 1054-1079.

Harrison, E. J., Jr., 1973: Three-dimensional numerical simulations of tropical systems utilizing nested fine grids. J. Atmos. Sci., 30, $1528-1543$.

Holland, G. J., 1980: An analytic model of the wind and pressure profiles in hurricanes. Mon. Wea. Rev., 108, 1212-1218.

Joint Typhoon Warning Center, 1988: Annual tropical cyclone report. U.S. Naval Oceanography Command Center, San Francisco, CA, 219 pp. [NTIS ADA207206.]

— http://www.npmocw.navy.mil/npmocw/prods/products/ best_tracks.]

Jones, R. W., 1977: A nested grid for a three-dimensional model of a tropical cyclone. J. Atmos. Sci., 34, 1528-1553.

Khain, A. P., and I. Ginis, 1991: The mutual response of a moving tropical cyclone and the ocean. Beitr. Phys. Atmos., 64, 125141

Kurihara, Y., and J. L. Holloway Jr., 1967: Numerical integration of a nine-level global primitive equations model formulated by the box method. Mon. Wea. Rev., 95, 509-530.

- and G. J. Tripoli, 1976: An iterative time integration scheme designed to preserve a low-frequency wave. Mon. Wea. Rev., 104, 761-764

- and M. A. Bender, 1980: Use of a movable nested-mesh model for tracking a small vortex. Mon. Wea. Rev., 108, 1792-1809.

— , G. J. Tripoli, and M. A. Bender, 1979: Design of a movable nested-mesh primitive equation model. Mon. Wea. Rev., 107, 239-249.

— , R. Tuleya, and M. A. Bender, 1998: The GFDL hurricane prediction system and its performance in the 1995 hurricane season. Mon. Wea. Rev., 126, 1306-1322.

Large, W. G., and S. Pond, 1981: Open ocean momentum flux measurements in moderate to strong winds. J. Phys. Oceanogr., 11, 324-336.

Larichev, V. D., and G. M. Reznik, 1976: Two-dimensional solitary Rossby waves. Dokl. Akad. Nauk SSSR, 231, 1077-1079.

Levitus, S., 1982: Climatological Atlas of the World Ocean. NOAA Professional Paper 13, U.S. Government Printing Office, Washington, DC, 173 pp. [Available from U.S. Government Printing Office, Washington, DC 20402.]

Ley, G. W., and R. L. Elsberry, 1976: Forecasts of Typhoon Irma using a nested-grid model. Mon. Wea. Rev., 104, 1154-1161.

NOAA/National Weather Service, 1979: Meteorological criteria for standard project hurricane and probable maximum hurricane wind fields, Gulf of Mexico and east coast of the United States. NOAA/National Weather Service Tech. Rep. 23, 320 pp. [NTIS PB80117997.

Oey, L.-Y., and P. Chen, 1992: A nested grid ocean model with application to the simulation of meanders and eddies in the Norwegian Coastal Current. J. Geophys. Res., 97, $20063-20086$.

Price, J. F., T. B. Sanford, and G. Z. Forristall, 1994: Forced stage response to a moving hurricane. J. Phys. Oceanogr., 24, 233260.

Schade, L. R., and K. A. Emanuel, 1999: The ocean's effect on the intensity of tropical cyclones: Results from a simple coupled atmosphere-ocean model. J. Atmos. Sci., 56, 642-651.

Smagorinsky, J., 1963: General circulation experiments with primitive equations: I. The basic experiment. Mon. Wea. Rev., 91, 99-164.

Spall, M. A., and W. R. Holland, 1991: A nested primitive equation model for oceanic application. J. Phys. Oceanogr., 21, 205-220. 